\title{
Controlling Singlet Fission by Molecular Contortion
}

Felisa S. Conrad-Burton[1] $]^{\mathrm{a}}$, Taifeng Liu[1] ${ }^{* \mathrm{a}, \mathrm{b}}$, Florian Geyer ${ }^{\mathrm{a}}$, Roberto Costantini ${ }^{\mathrm{a}, \mathrm{d}, \mathrm{e}}$, Andrew P. Schlaus $^{\mathrm{a}}$, Michael S. Spencer ${ }^{\mathrm{a}}$, Jue Wang ${ }^{\mathrm{a}}$, Raul Hernández Sánchez ${ }^{\mathrm{a}}$, Boyuan Zhang ${ }^{\mathrm{a}}$, Qizhi Xu ${ }^{\mathrm{a}, \mathrm{c}}$, Michael L. Steigerwald ${ }^{\mathrm{a}}$, Shengxiong Xiao ${ }^{\mathrm{b}}$, Hexing Li ${ }^{\mathrm{b}}$ Colin P. Nuckolls*a,b Xiaoyang Zhu*a

\section{TABLE OF CONTENTS}

\author{
1. Synthesis Details and Molecular Dynamics \\ 2. Crystal Structure Characterization \\ 3. Steady-State Absorption and Emission Spectra \\ 4. Electrochemistry and Energy Levels \\ 5. Geometry and Molecular Orbitals from Calculations \\ 6. Sample Preparation \\ 7. Ultrafast Spectroscopy \\ 8. Computational Details and Crystal Data
}

\section{General Information}

Unless otherwise noted, all materials and reagent including dry solvents were obtained from commercial suppliers and used without further purification. Perylene tetracarboxydiimide were prepared according to the procedures reported in the literature. $^{1,} 2$ Unless otherwise noted, all work-up processing and purification procedures were carried out with reagent-grade solvents in air.

${ }^{1} \mathrm{H}$, and ${ }^{13} \mathrm{C}$ NMR spectra were obtained from a Bruker DRX300 (300 MHz), Bruker DRX400 (400 MHz) or a Bruker DMX500 (500 MHz) spectrometer. The mass spectroscopic and HRMS data were obtained at the Columbia University mass spectrometry facility using a Bruker ultrafleXtreme MALDI TOF/TOF. Absorption 
spectra were obtained on Shimadzu UV 1800 UV-Vis spectrophotometer. Cyclic voltammograms (CVs) were recorded on a $\mathrm{CH} 166$ electrochemical workstation using $\mathrm{Ag} / \mathrm{AgCl}$ electrode as the reference electrode and $0.1 \mathrm{M}$ tetrabutylammonium hexafluorophosphate $\left(\mathrm{TBAPF}_{6}\right)$ in tetrahydrofuran as the supporting electrolyte. All cyclic voltammetry data was referenced to the known ferrocene/ferrocenium couple. The X-ray diffraction (XRD) patterns were recorded on a Bruker X-ray diffraction instrument using $\mathrm{Cu} \mathrm{K \alpha}$ radiation.

All quantum mechanical calculations were performed using Jaguar, version 8.2, Schrodinger, Inc., New York, NY, 2013. (See A. D. Bochevarov, E. Harder, T. F. Hughes, J. R. Greenwood, D. A. Braden, D. M. Philipp, D. Rinaldo, M. D. Halls, J. Zhang, R. A. Friesner, "Jaguar: A High Performance Quantum Chemistry Software Program with Strengths in Life and Materials Sciences", Int. J. Quantum Chem., 2013, 113(18), 2110 2142). All geometries were optimized using the B3LYP functional and the $6-31 \mathrm{G}^{* *}$ basis set.

\section{Synthesis Details and Molecular Dynamics}

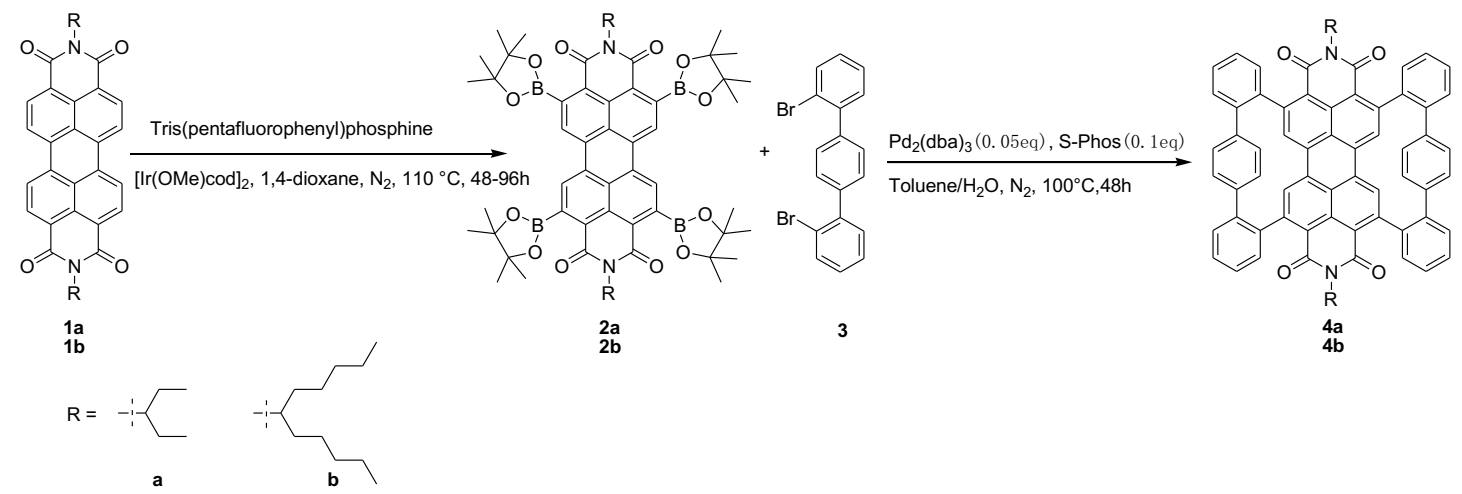

Scheme 1. Synthesis route towards PDI-terphenyl bowl-shaped molecule (PDI-B) 4ab with different alkyl chains

\section{Compound 1a, 1b and 1c}

These starting materials $\mathbf{1 a}, \mathbf{1 b},{ }^{3-5}$ 1,4-bis(2-bromophenyl)benzene $\mathbf{3}^{6}$ were synthesized according to the literatures previously reported.

Synthesis of N,N'-Bis(alkyl)-2,5,8,11-tetra(4,4,5,5-tetramethyl-1,3,2- 
dioxaborolan-2-yl)- perylene-3, 4:9,10-tetracarboxylic acid bisimide 2.

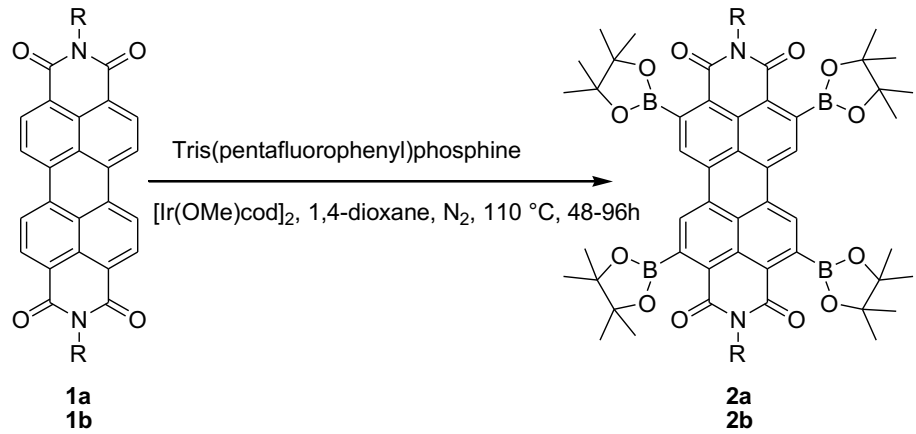

The molecules $\mathbf{2 a}$ and $\mathbf{2 b}$ were synthesized following the procedure previously reported by Hiroshi Shinokubo group. ${ }^{7}$ To a Schlenk flask was added 2 (2 mmol), [ $\mathrm{Ir}(\mathrm{OMe}) \operatorname{cod}_{2}$ (40 $\mathrm{mg}, 0.06 \mathrm{mmol})$, tris(pentafluorophenyl)phosphine (128 mg, 0.24mol), and bis(pinacolato)diboron ( $4.06 \mathrm{~g}, 16 \mathrm{mmol})$. This flask was evacuated and backfilled with $\mathrm{N}_{2}$ three times, then $40 \mathrm{~mL}$ of anhydrous 1,4-dioxane was added under $\mathrm{N}_{2}$ atmosphere. The mixture was stirred under nitrogen at $110^{\circ} \mathrm{C}$ for $2-4$ days. The solvent of reaction mixture was removed by rotatory evaporator, and the residue was filtered quickly through silica gel plug by using dichloromethane as eluent. Then the solvent was removed and the residue was recrystallized from $\mathrm{CH}_{2} \mathrm{Cl}_{2} / \mathrm{MeOH}$, resulting in 2 with 60 $70 \%$ yield.

Compound 2a: ${ }^{1} \mathrm{H}$ NMR (400 MHz, $\left.\mathrm{CDCl}_{3}\right) \delta 8.52(\mathrm{~s}, 4 \mathrm{H}), 4.95-4.87(\mathrm{~m}, 2 \mathrm{H}), 2.23-$ $2.12(\mathrm{~m}, 4 \mathrm{H}), 1.99-1.89(\mathrm{~m}, 4 \mathrm{H}), 1.54(\mathrm{~s}, 48 \mathrm{H}), 0.94-0.90(\mathrm{t}, 12 \mathrm{H}) ;{ }^{13} \mathrm{C} \mathrm{NMR}(126 \mathrm{MHz}$, $\left.\mathrm{CDCl}_{3}\right) \delta 165.80,138.48,133.18,128.09,126.74,125.81,84.48,58.02,25.04,11.62$; ESI HRMS (m/z): Calcd. for $[\mathrm{M}+\mathrm{H}]^{+} 1035.5725$; Found: $[\mathrm{M}+\mathrm{H}]^{+} 1035.5718$.

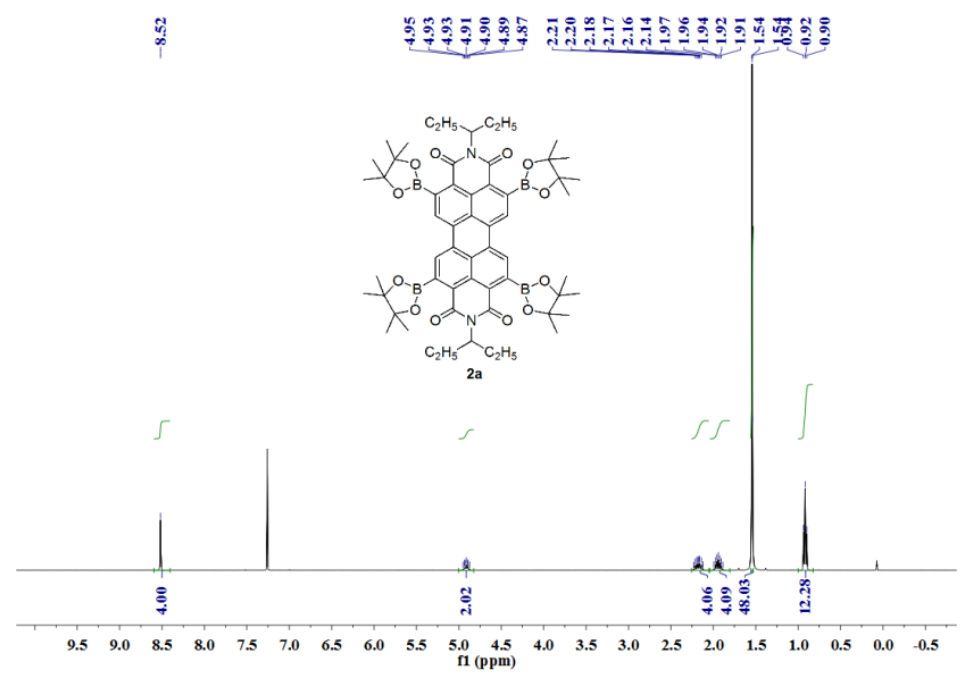




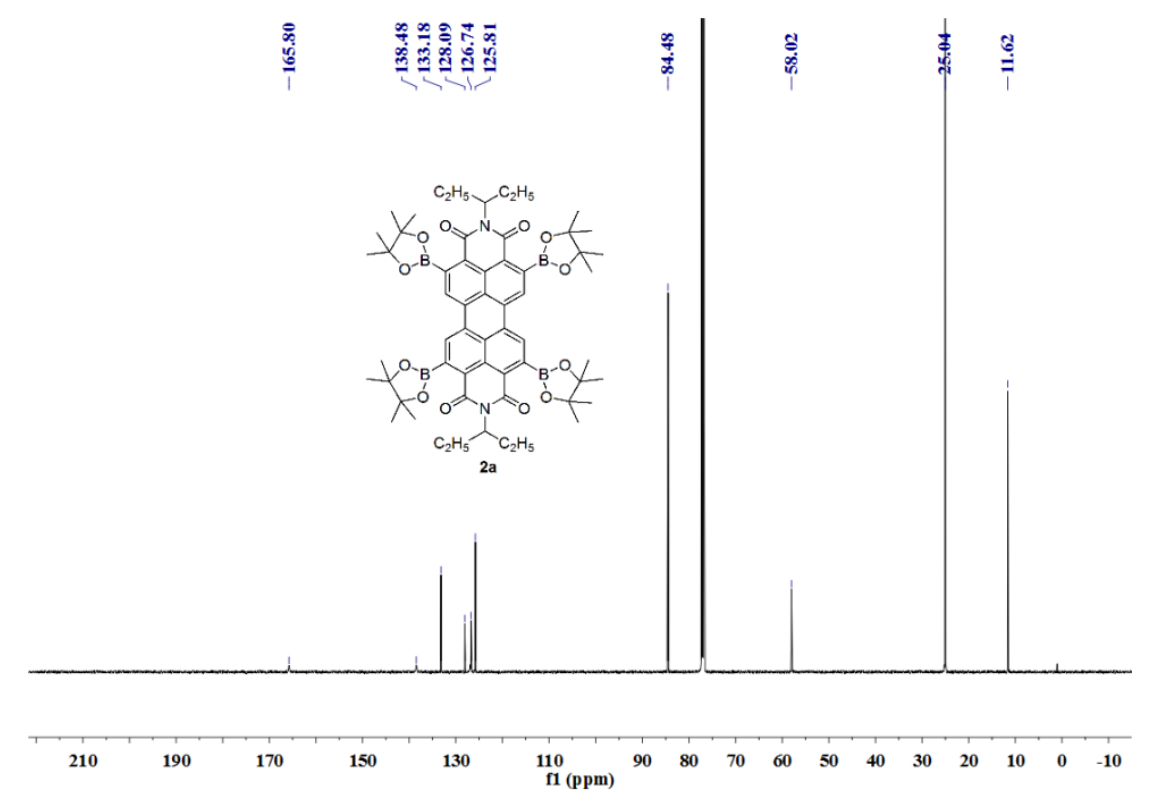

Compound 2b: ${ }^{1} \mathrm{H}$ NMR $\left(500 \mathrm{MHz}, \mathrm{CDCl}_{3}\right) \delta 8.51(\mathrm{~s}, 4 \mathrm{H}), 5.07-5.01(\mathrm{~m}, 2 \mathrm{H}), 2.20-$ $2.16(\mathrm{~m}, 4 \mathrm{H}), 1.85-1.81(\mathrm{~m}, 4 \mathrm{H}), 1.54(\mathrm{~s}, 48 \mathrm{H}), 1.29-1.25(\mathrm{~m}, 24 \mathrm{H}), 0.86-0.83(\mathrm{t}, 12 \mathrm{H})$; ${ }^{13} \mathrm{C}$ NMR $\left(126 \mathrm{MHz}, \mathrm{CDCl}_{3}\right) \delta 165.94,165.53,138.78,133.32,128.19,126.88,125.93$, 84.60, 54.89, 32.40, 31.85, 26.67, 25.19, 22.66, 14.23; ESI HRMS (m/z): Calcd. for $[\mathrm{M}+\mathrm{H}]^{+}$1203.7627; Found: $[\mathrm{M}+\mathrm{H}]^{+} 1203.7619$.

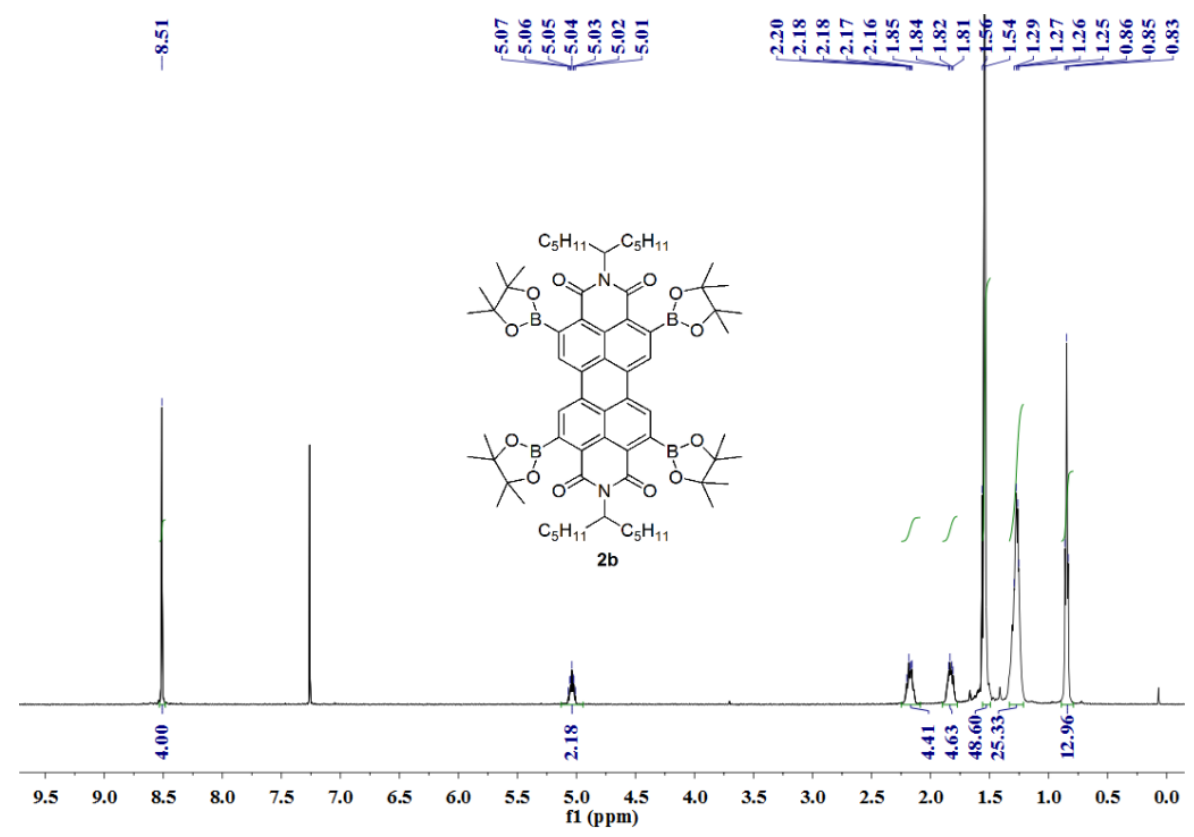




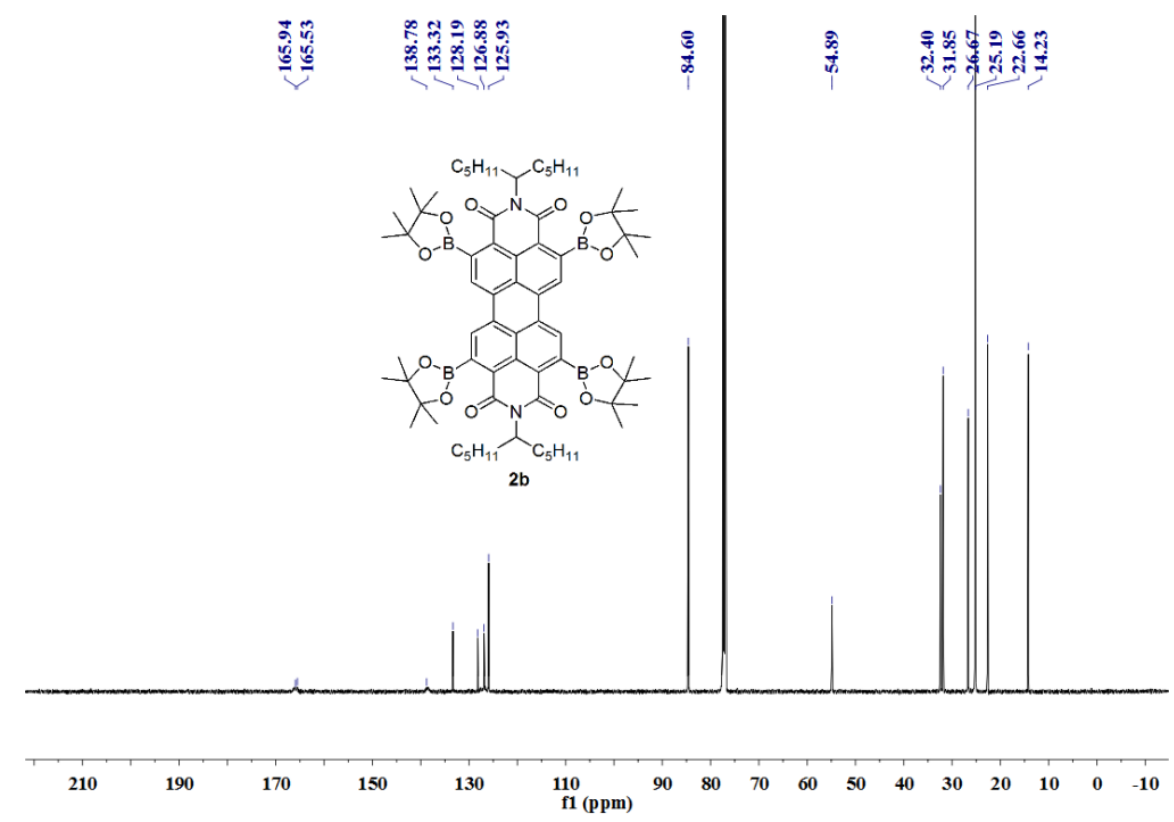

\section{Synthesis of 1,4-bis(2-bromophenyl)benzene $3^{6}$}

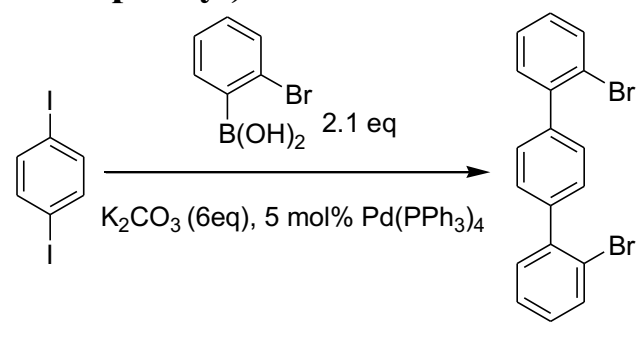

3

This terphenyl dibromide was synthesized following the procedure previously reported by Alexandra Velian and coworkers. ${ }^{6}$ To a Schlenk flask, 1,4-diiodobenzene (3.30 g, $10.0 \mathrm{mmol}$ ), 2-bromo-phenylboronic acid (4.22 g, $21 \mathrm{mmol}, 2.1$ equiv), $\mathrm{K}_{2} \mathrm{CO}_{3}(8.28 \mathrm{~g}$, 60 mmol, 6 equiv), and $\mathrm{Pd}\left(\mathrm{PPh}_{3}\right)_{4}(578 \mathrm{mg}, 0.5 \mathrm{mmol}, 0.05$ equiv) was added and evacuated and backfilled with $\mathrm{N}_{2}$ for three times. Then, $200 \mathrm{~mL}$ toluene, $50 \mathrm{~mL}$ ethanol and $50 \mathrm{~mL}$ water were added into the flask under nitrogen protection. The mixture was stirred for $36 \mathrm{~h}$ at $65^{\circ} \mathrm{C}$ under $\mathrm{N}_{2}$ protection. When cooled to room temperature, solvent was removed via rotary evaporation. This mixture was extracted with $\mathrm{CH}_{2} \mathrm{Cl}_{2}$. The combined organic phase was dried by $\mathrm{Na}_{2} \mathrm{SO}_{4}$, and concentrated via rotary evaporation. The residue was recrystallized from $\mathrm{CH}_{2} \mathrm{Cl}_{2} / \mathrm{MeOH}$, resulting to pale yellow solid 2.8 g (60\% yield, $11.07 \mathrm{mmol}) .{ }^{1} \mathrm{H}$ NMR $\left(400 \mathrm{MHz}, \mathrm{C}_{6} \mathrm{D}_{6}\right) \delta$ 7.50-7.47 (dd, 2H), 7.39(s, $4 \mathrm{H}), 7.08-7.06$ (dd, 2H), 6.95-6.91 (td, 2H), 6.76-6.72 (td, 2H); ${ }^{13} \mathrm{C}$ NMR (101 MHz, $\left.\mathrm{C}_{6} \mathrm{D}_{6}\right) \delta 142.76,140.79,133.51,131.71,129.46,129.01,127.54,123.05$; TOF ASAP $^{+}$ MS (m/z): Calcd. For $[\mathrm{M}]^{+}$385.9286; Found: $385.9292[\mathrm{M}]^{+}$. 


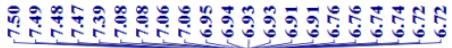

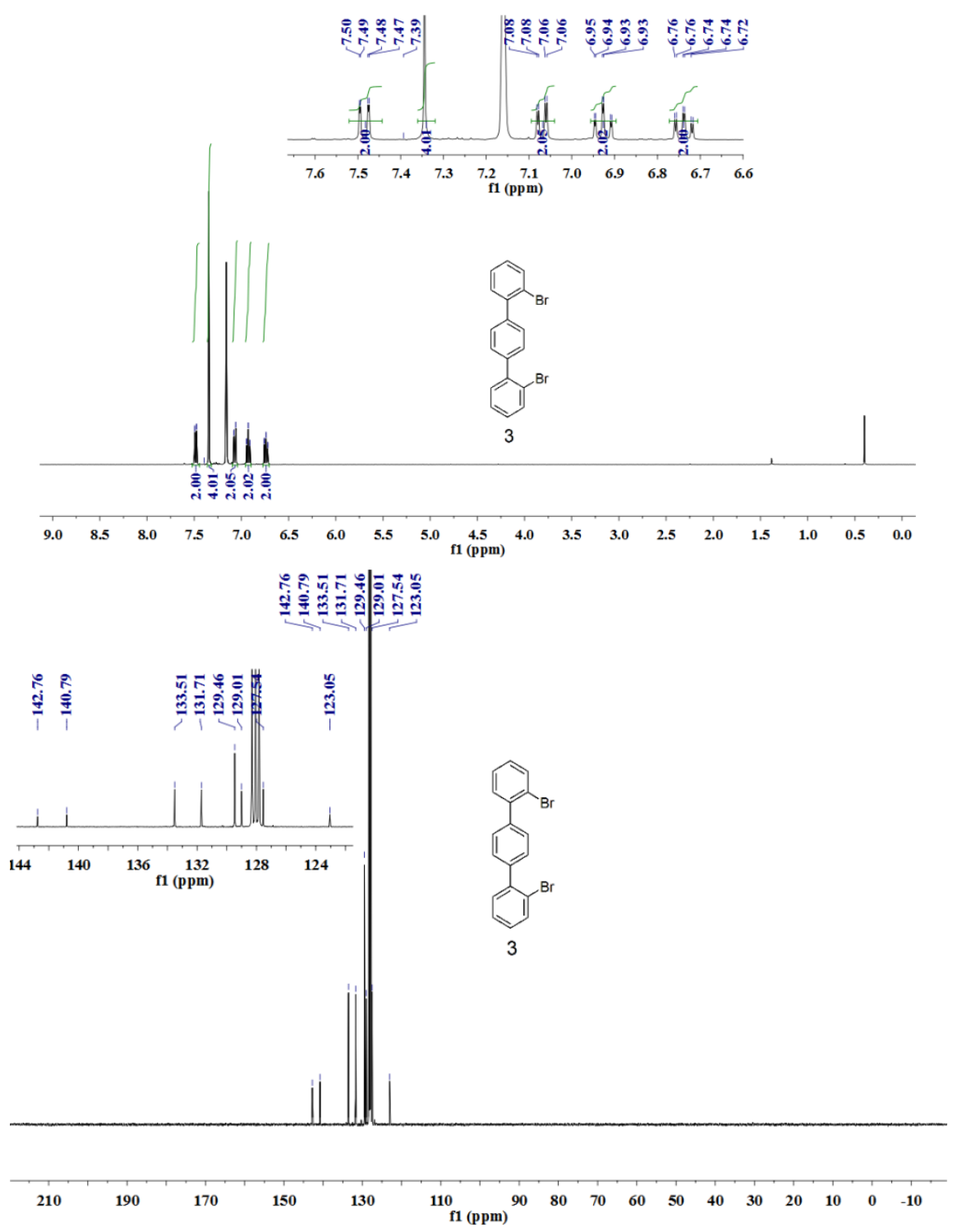

\section{Synthesis of PDI terphenyl bowl 4}
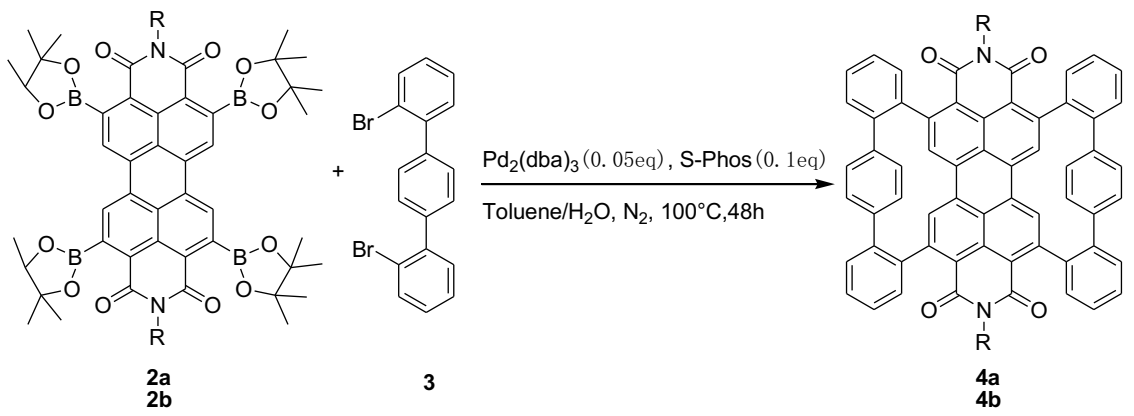

These PDI-terphenyl bowls were synthesized by Suzuki-cross coupling reaction using Sphos and $\mathrm{Pa}_{2}(\mathrm{dba})_{3}$ as catalyst system. Compound 2 (0.1 mmol, 1.0 eq), 1,4-bis(2- 
bromophenyl)benzene 3 (77.6 mg, $0.2 \mathrm{mmol}, 2.0$ eq), $\mathrm{K}_{2} \mathrm{CO}_{3}$ (276 mg, $2.0 \mathrm{mmol}, 20.0$ equiv), $\mathrm{Pa}_{2}(\mathrm{dba})_{3}$ (3.7 mg, $0.004 \mathrm{mmol}, 0.04$ equiv) and Sphos ( $4.1 \mathrm{mg}, 0.01 \mathrm{mmol}, 0.1$ equiv) were added to a $100 \mathrm{~mL}$ Schlenk flask. Then, this flask was evacuated and backfilled with $\mathrm{N}_{2}$ for three times. The solution of $50 \mathrm{~mL}$ toluene and $5 \mathrm{~mL}$ water was bubbled for $30 \mathrm{~min}$ then was transfered into the flask under nitrogen pressure. The mixture was stirred for $60 \mathrm{~h}$ at $100{ }^{\circ} \mathrm{C}$ under $\mathrm{N}_{2}$ protection. When cooled to room temperature, the organic phase was dried by $\mathrm{Na}_{2} \mathrm{SO}_{4}$, and concentrated via rotary evaporation. The residue was purified by silica gel chromatography column with dichloromethane/hexane as eluent. The main products were recrystallized from $\mathrm{CH}_{2} \mathrm{Cl}_{2} / \mathrm{MeOH}$, resulting to dark red solid 10-15 mg (10-15\% yield).

Compound 4a: ${ }^{1} \mathrm{H}$ NMR (400 MHz, $\left.\mathrm{CDCl}_{3}\right) \delta$ 7.78, 7.76, 7.60, 7.58, 7.57, 7.40, 7.38, 7.36, 7.35, 7.33, 7.06, 6.91, 4.76, 4.74, 4.72, 4.71, 4.69, 1.97, 1.96, 1.94, 1.92, 1.78, $1.77,1.75,1.73,1.71,0.88,0.86,0.84 ;{ }^{13} \mathrm{C} \mathrm{NMR}\left(101 \mathrm{MHz}, \mathrm{CDCl}_{3}\right) \delta 163.20,145.53$, $139.71,139.66,136.22,134.33,133.54,132.87,132.44,130.14,129.43,129.22$, 127.93, 126.86, 125.98, 118.92, 57.71, 29.71, 24.53, 11.29; MALDI-TOF HRMS: Calcd. for $\mathrm{C}_{70} \mathrm{H}_{50} \mathrm{~N}_{2} \mathrm{O}_{4}(\mathrm{~m} / \mathrm{z})$ : 982.3771 ; Found: 982.3775 .

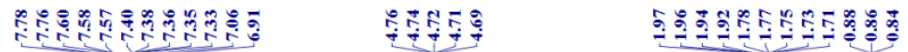
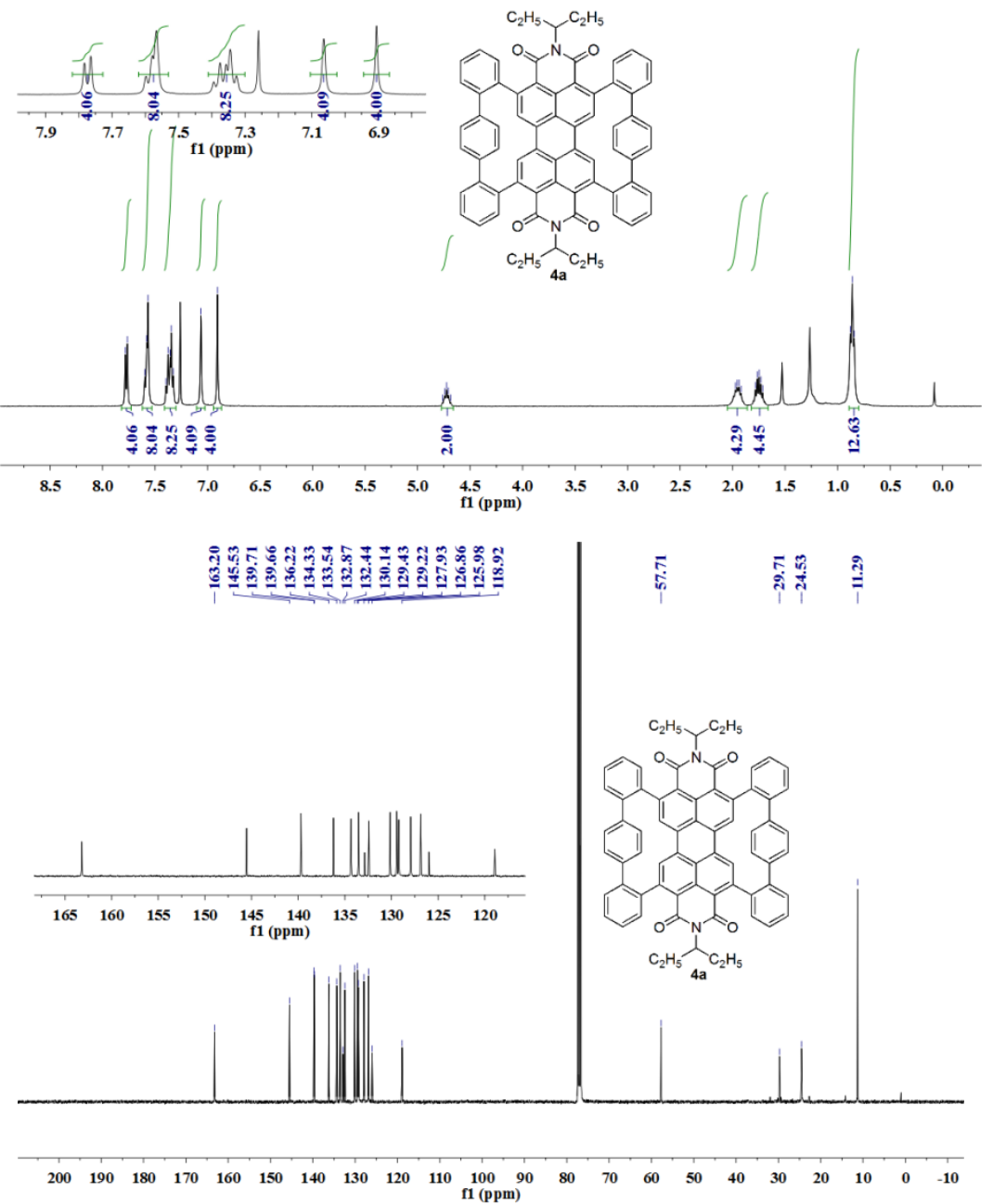
Compound 4b: ${ }^{1} \mathrm{H}$ NMR $\left(400 \mathrm{MHz}, \mathrm{CDCl}_{3}\right) \delta$ 7.80-7.78(d, 4H), 7.61-7.57(t, 4H; s, 4H), 7.44, 7.43, 7.42, 7.42, 7.39, 7.39, 7.37, 7.36, 7.35, 7.10, 7.00, 4.88, 4.85, 4.84, 4.82, 4.80, 1.95, 1.68, 1.26, 0.86; ${ }^{13} \mathrm{C}$ NMR $\left(126 \mathrm{MHz}, \mathrm{CDCl}_{3}\right) \delta 163.12,145.50,139.77$, $139.67,136.30,134.32,133.61,132.88,132.47,130.19,129.44,129.26,128.21$, $128.00,126.88,126.06,119.04,54.84,31.87,26.59,22.68,14.17$; MALDI-TOF HRMS: Calcd. for $\mathrm{C}_{82} \mathrm{H}_{74} \mathrm{~N}_{2} \mathrm{O}_{4}(\mathrm{~m} / \mathrm{z}) 1150.5649$; found 1150.5646 .

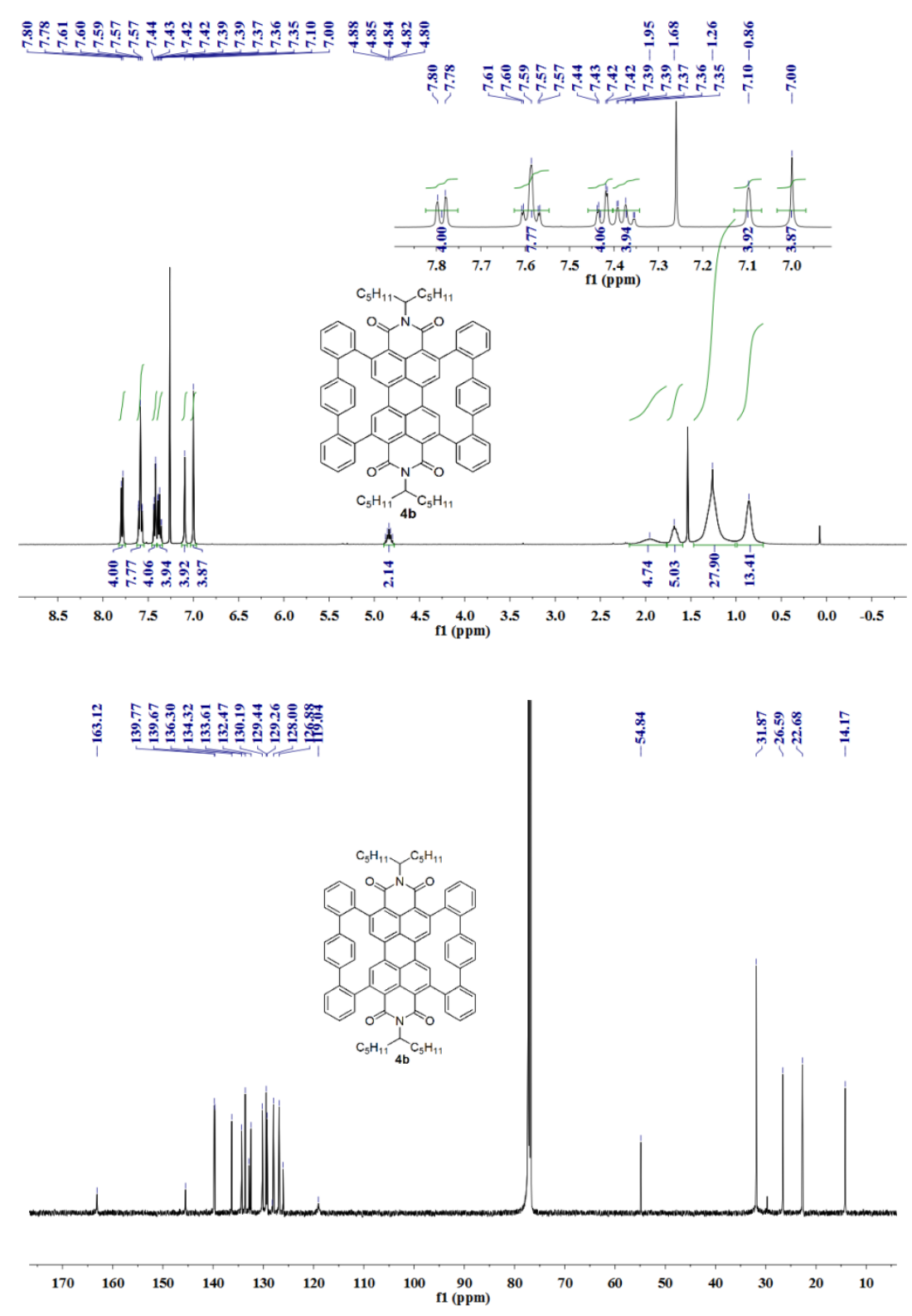




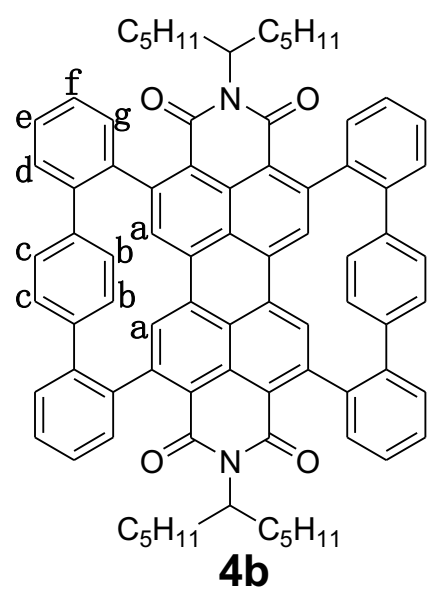

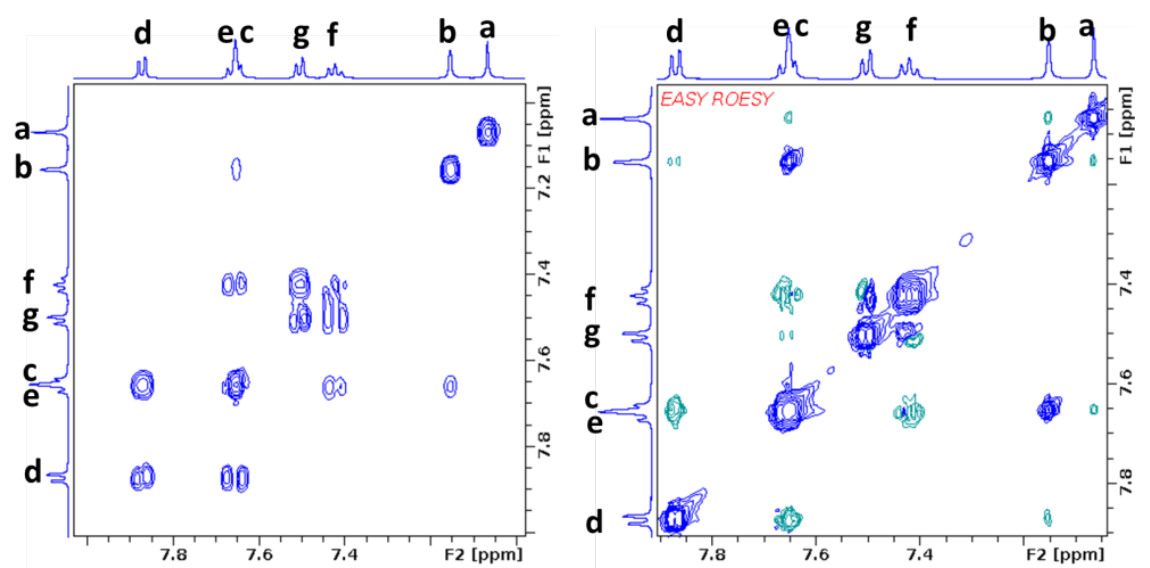

Figure S1. a) $2 \mathrm{D}{ }^{1} \mathrm{H}-{ }^{1} \mathrm{H}$ COSY spectrum of PDI bowl $\mathbf{4 b}$ in tetrachloroethane- $d_{2}(500$ $\mathrm{MHz}, 300 \mathrm{~K})$; b) 2D ${ }^{1} \mathrm{H}-{ }^{1} \mathrm{H}$ EASY-ROESY spectrum of PDI bowl $\mathbf{4 b}$ in tetrachloroethane- $d_{2}(500 \mathrm{MHz}, 300 \mathrm{~K})$.

To attribute all protons of PDI-B $\mathbf{4 b}$ at aromatic region, ${ }^{1} \mathrm{H}-{ }^{1} \mathrm{H}$ COSY and EASY ROESY spectra were performed (Figure S1). Singlet protons $\mathrm{H}^{\mathrm{a}}$ at $7.07 \mathrm{ppm}$ have no coupling interaction with other protons in COSY spectrum (Figure S1 a) but have longrange weak coupling interaction with $\mathrm{H}^{\mathrm{b}}$ and $\mathrm{H}^{\mathrm{c}}$ in EASY ROESY spectrum (Figure $\mathrm{S} 1$ b). $\mathrm{H}^{\mathrm{a}}$ is attributed to PDI bay-protons in the strong shielding circumstance generated by the mid-phenyl of terphenyl. $\mathrm{H}^{\mathrm{b}}$ at $7.15 \mathrm{ppm}$ and $\mathrm{H}^{\mathrm{c}}$ (submerged in triplet $\mathrm{H}^{\mathrm{e}}$ ) at 7.65 ppm, each integrating to $4 \mathrm{H}$, is assigned to the mid phenyl of terphenyl. $\mathrm{H}^{\mathrm{b}}$ impacted by the shielding effect from the PDI bowl, faces to the inner of the bowl and shifts to upfield compared with $\mathrm{H}^{\mathrm{c}}$. Notably, the strong long-range coupling between $\mathrm{H}^{\mathrm{b}}$ and $\mathrm{H}^{\mathrm{c}}$ in EASY ROESY spectrum (Figure S1 b) exhibits the central phenyl can swing but cannot rotate freely at $300 \mathrm{~K}$. The attribution of other protons is identified along with the aid of EASY-ROESY NMR spectra. $\mathrm{H}^{\mathrm{d}}$ has strong $\mathrm{H}-\mathrm{H}$ coupling with $\mathrm{H}^{\mathrm{c}}$ and $\mathrm{H}^{\mathrm{e}}$, which supports $\mathrm{H}^{\mathrm{d}}$ closes to mid-phenyl and triplet $\mathrm{H}^{\mathrm{e}}$. The strong links between $\mathrm{H}^{\mathrm{f}}$ and $\mathrm{H}^{\mathrm{e}}, \mathrm{H}^{\mathrm{f}}$ and $\mathrm{H}^{\mathrm{g}}$, help to attribute $\mathrm{H}^{\mathrm{g}}$ to the protons close to carboxyl of PDI. 

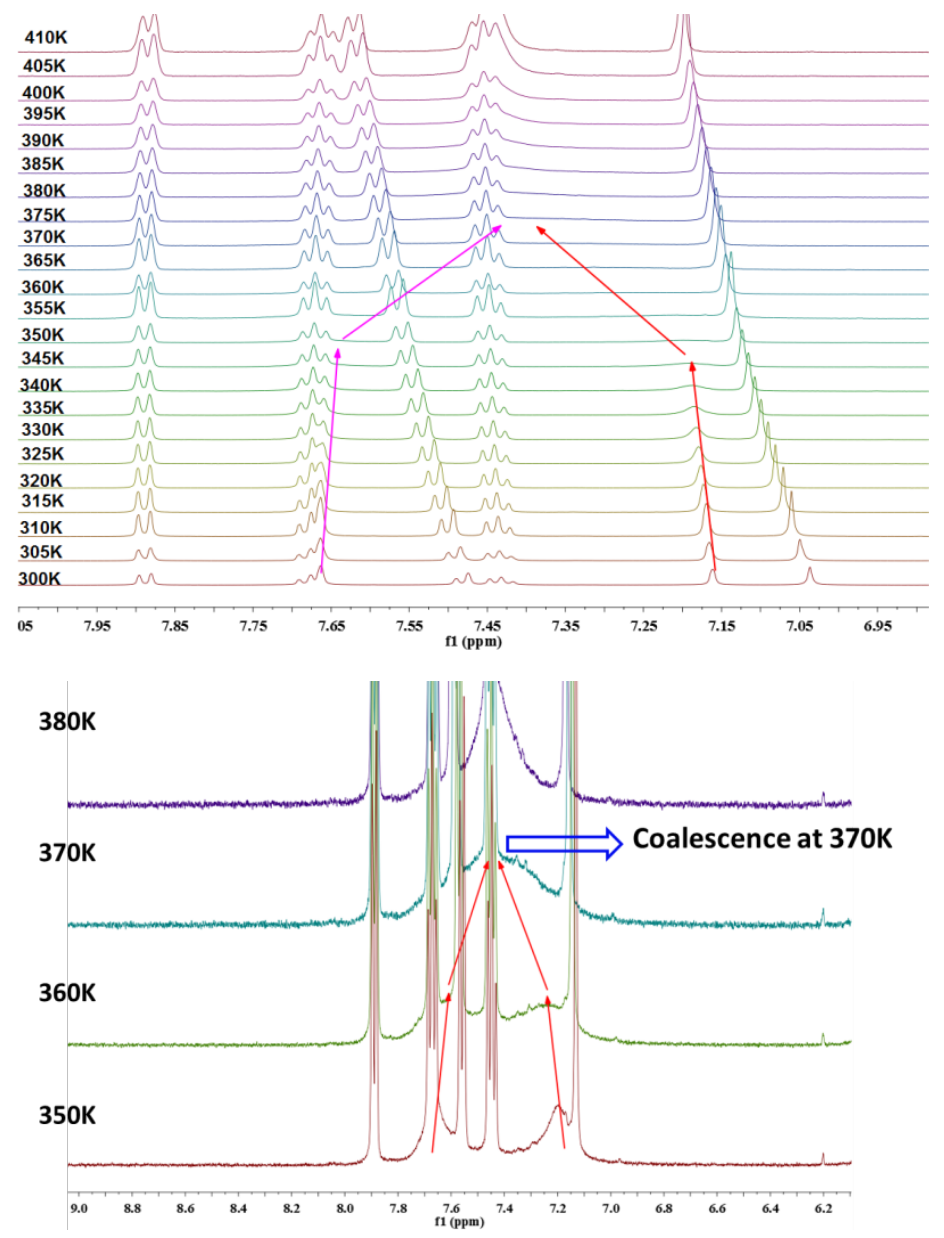

Figure S2. Variable temperature (V-T) ${ }^{1} \mathrm{HNMR}(500 \mathrm{~Hz})$ of PDI-B $4 \mathbf{b}$ in tetrachloroethane- $\mathrm{d}_{2}$ under air from $300 \mathrm{~K}$ to $410 \mathrm{~K}$ (up); the enlarged view in the range of 6 - 9 ppm from $350 \mathrm{~K}$ to $380 \mathrm{~K}$ (down).

To test stability and dynamics of PDI-B in solvent under air, variable temperature (V-T) ${ }^{1} \mathrm{HNMR}$ was performed. The coalescence at $370 \mathrm{~K}$ demonstrates the central phenyl of terphenyl can rotate freely above $370 \mathrm{~K}$, which also exhibits the extraordinary stability of PDI-B in air.

Table S1. Rotation activation parameters of central phenyl in terphenyl in PDI-B $\mathbf{4 b}$ (All raw data come from the V-T NMR in Figure S2).

The central phenyl rotation rates values $\mathrm{Kr}$ were estimated by using the Topspin DNMR module to fit the line shape in variable experimental spectra per $5 \mathrm{~K}$ from 315 $\mathrm{K}$ to $350 \mathrm{~K}$. Above $350 \mathrm{~K}$, the peak was too broad and below $315 \mathrm{~K}$ there was not sufficient broadening. To fit the spectra, we only fitted the upfield peak of central phenyl at $7.18 \mathrm{ppm}$, and varied its shift and exchange rate. The shift of another exchanging peak was fixed at $7.663 \mathrm{ppm}$ for all spectra. An important parameter is the $\mathrm{lb}$ (line broadening value) and that was determined by first fitting the solvent peak for each spectrum. 


\begin{tabular}{|c|c|c|c|c|}
\hline Temp/K & $\begin{array}{c}\text { Shift } \\
\text { 1/ppm }\end{array}$ & $\mathbf{K r} / \mathrm{Hz}$ & $\mathbf{l b}$ & $\begin{array}{c}\text { Shift } \\
\mathbf{2} / \mathrm{nm}\end{array}$ \\
\hline 315 & 7.663 & 8.1 & $1.416(\mathrm{D})$ & 7.1728 \\
\hline 320 & 7.663 & 10.8 & $1.55(\mathrm{D})$ & 7.1762 \\
\hline 325 & 7.663 & 15.7 & $1.7(\mathrm{D})$ & 7.1792 \\
\hline 330 & 7.663 & 23.7 & $1.8(\mathrm{D})$ & 7.1819 \\
\hline 335 & 7.663 & 39 & $2.16(\mathrm{D})$ & 7.1842 \\
\hline 340 & 7.663 & 63.6 & $2.2(\mathrm{D})$ & 7.1855 \\
\hline 345 & 7.663 & 109 & $2.2(\mathrm{D})$ & 7.1834 \\
\hline 350 & 7.663 & 169 & $2.34(\mathrm{D})$ & 7.1766 \\
\hline
\end{tabular}

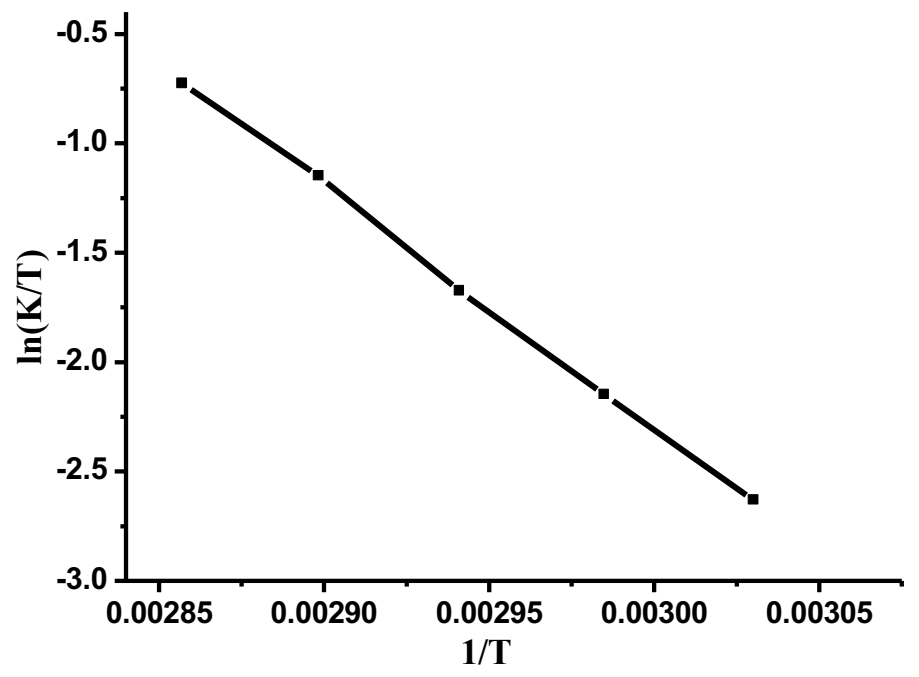

Figure S3: Plot of $\ln (\mathrm{Kr} / \mathrm{T})$ vis $1 / \mathrm{T}$ according to the Eyring equation $\ln (\mathrm{Kr} / \mathrm{T})=-$ $\Delta \mathrm{H}^{\ddagger} / \mathrm{RT}+\left[\ln \left(\mathrm{k}_{\mathrm{b}} / \mathrm{h}+\Delta \mathrm{S}^{\ddagger}\right)\right]$.

The mid-phenyl rotation rates in terphenyl were estimated by using the DNMR module within Topspin software per $5 \mathrm{~K}$ from $315 \mathrm{~K}$ to $350 \mathrm{~K}$ (Figure S3). Then, Eyring plot gives the mid-phenyl rotation activation parameters $\Delta \mathrm{H}^{\ddagger}=18.36 \mathrm{kcal} \mathrm{mol}^{-1}$, $\Delta \mathrm{S}^{\ddagger}=3.01 \mathrm{kcal} \mathrm{mol}^{-1}$ and $\Delta \mathrm{G}^{\ddagger}=17.25 \mathrm{kcal} \mathrm{mol}^{-1}$.

\section{Crystal Structure Characterization}

The X-ray quality crystal of PDI-B 4a was grew by slowly diffusing acetonitrile into the solution of $\mathbf{4 a}$ in 1,4-dioxane and chloroform (v/v, 1:1). The X-ray diffraction analysis of $\mathbf{4 a}$ exhibits PDI-B 4a with a bow-shaped conformation, constructed from one bent PDI and two bent terphenyl linkers (Figure S4-7). PDI-B 4a in crystal shows a dimer-like stacking, in which a PDI-B dimer adopts a back-to-back pi-pi stacking. 


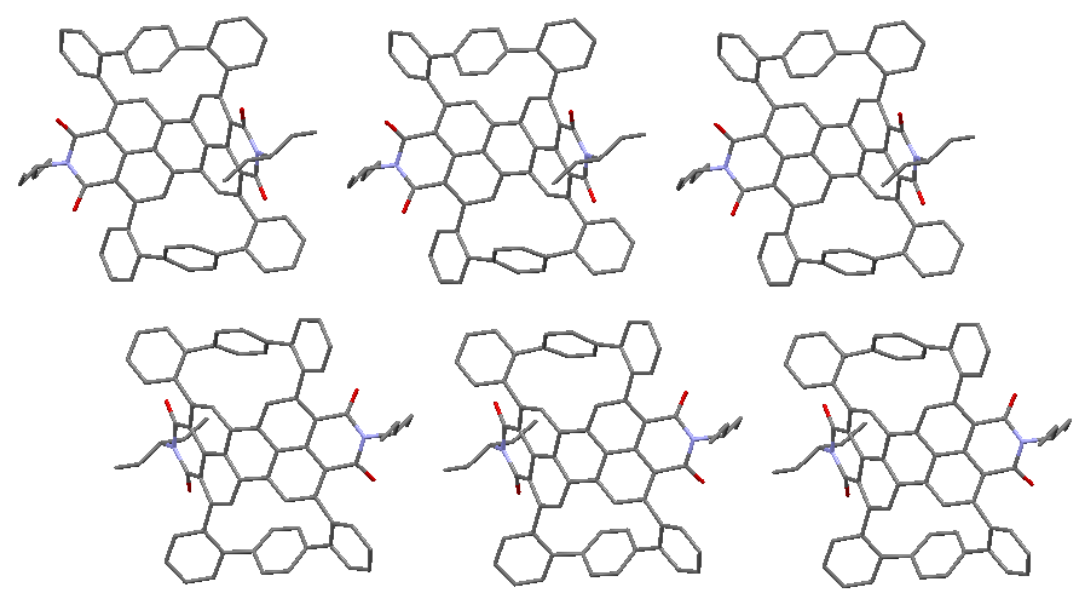

Figure S4: Stacking of PDI-B 4a along a-axis
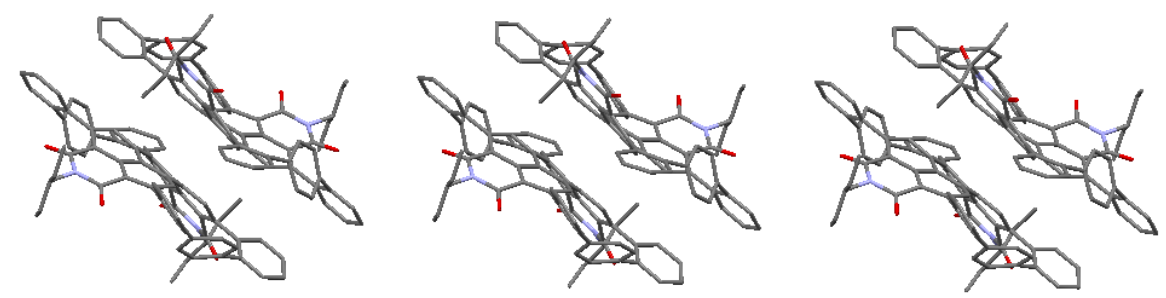

Figure S5: Stacking of PDI-B 4a along b-axis. This is the only axis with pi-pi interactions. 

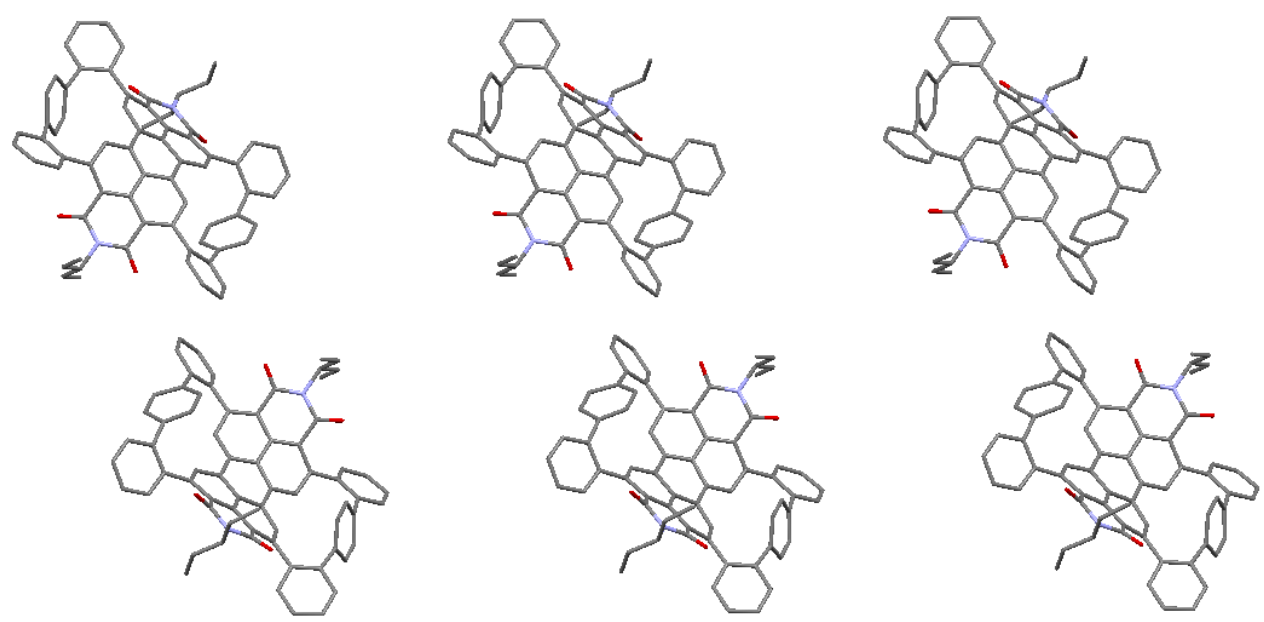

Figure S6: Stacking of PDI-B 4a along c-axis

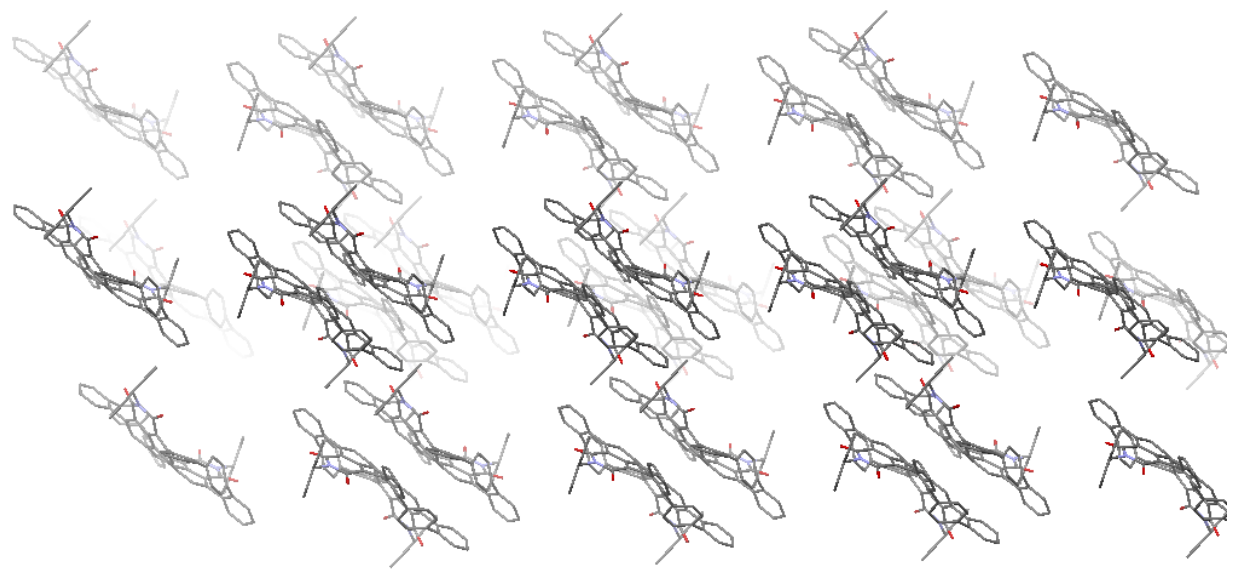

Figure S7: Stacking of PDI-B 4a in depth cueing 3D view.

\section{Steady-State Absorption and Emission Spectra}

UV-Visible Absorbance: All steady-state UV-vis absorption spectra were recorded by a Shimadzu UV-1800 spectrophotometer.

Fluorescence: PL data from Figure 1 of the main text were taken with a Horiba Scientific Fluoromax 4 fluorimeter. PDI was excited at $526 \mathrm{~nm}$ and PDI-B was excited at $590 \mathrm{~nm}$. 


\section{Electrochemistry and Energy Levels}

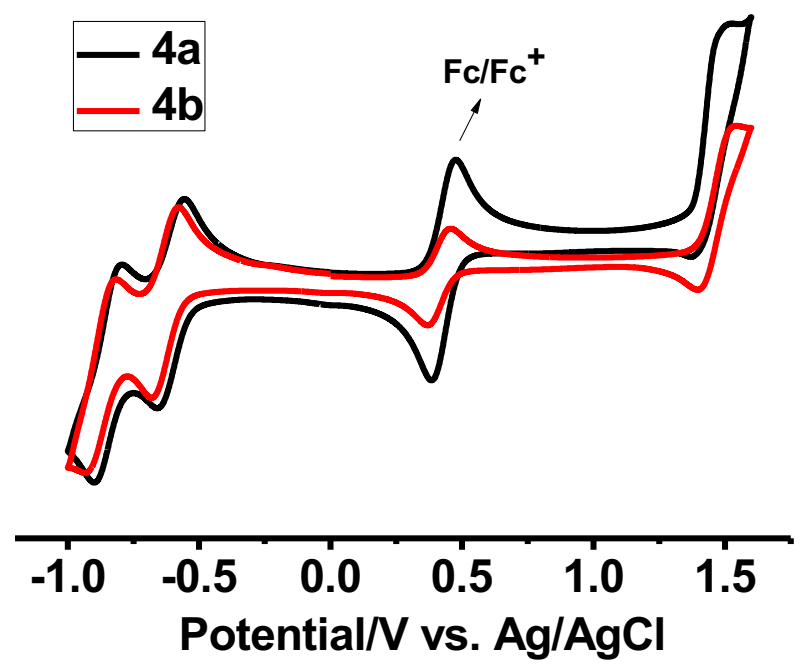

Figure S8 Cyclic voltammetry of PDI-B $4 \mathbf{a}$ and $\mathbf{4 b}\left(0.1 \mathrm{M} n-\mathrm{Bu}_{4} \mathrm{NPF}_{6}\right.$ in DCM $)$ at a scan rate of $100 \mathrm{mV} \mathrm{s}^{-1}$.

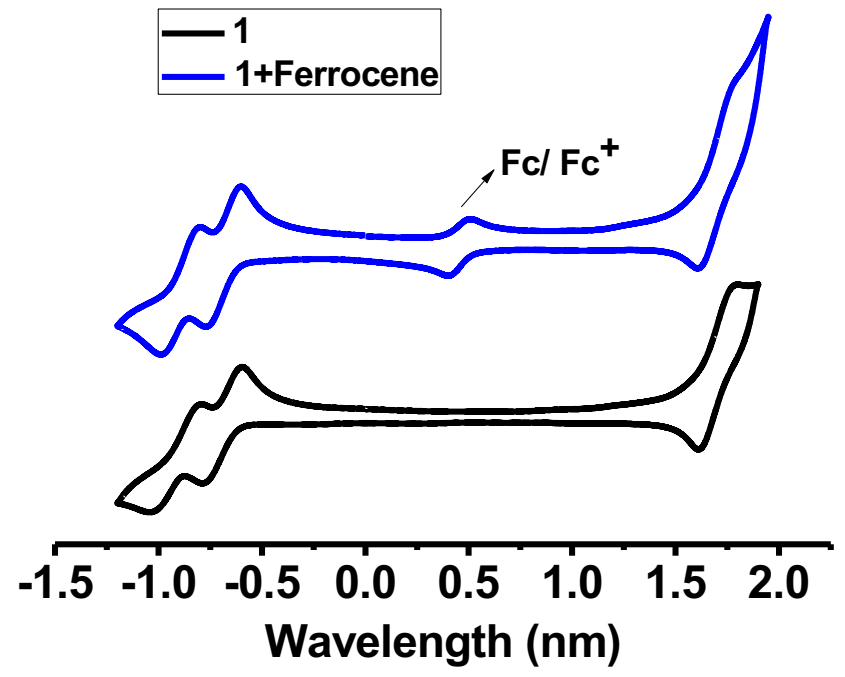

Figure S9 Cyclic voltammetry of PDI monomer 1a was measured at a scan rate of 100 $\mathrm{mV} \mathrm{s}^{-1}$ in dichloromethane solution with $\mathrm{Bu}_{4} \mathrm{NPF}_{6}(0.1 \mathrm{M})$ as supporting electrolyte, $\mathrm{Ag} / \mathrm{AgCl}$ as reference electrode and the $\mathrm{Fc} / \mathrm{Fc}^{+}$redox couple as an internal standard. HOMO and LUMO energy levels and energy gap were calculated by the equations $\mathrm{E}_{\mathrm{HOMO}}=-\left[\mathrm{E}_{\mathrm{ox}}-\mathrm{E}_{\left(\mathrm{Fc} / \mathrm{Fc}^{+}\right.}{ }^{+}+4.8\right] \mathrm{eV}, \mathrm{E}_{\mathrm{LUMO}}=-\left[\mathrm{E}_{\mathrm{red}}-\mathrm{E}_{\left(\mathrm{Fc} / \mathrm{Fc}^{+}\right)}+4.8\right] \mathrm{eV}$ and $\mathrm{E}_{\mathrm{gap}}=\mathrm{E}_{\mathrm{LUMO}}-$ Еномо.

Table S2. Computational, optical and cyclic voltammetry data for $\mathbf{1}, \mathbf{4 a}$ and $\mathbf{4 b}$. 


\begin{tabular}{ccccccccc} 
& \multicolumn{3}{c}{ computational $^{\mathrm{a}}$} & \multicolumn{3}{c}{ optical $^{\mathrm{b}}$} & \multicolumn{3}{c}{ cyclic voltammetry $^{\mathrm{c}}$} \\
\cline { 2 - 8 } & $\mathrm{E}_{\text {HOMO }}$ & $\mathrm{E}_{\mathrm{LUMO}}$ & $\mathrm{E}_{\text {gap }}$ & $\lambda_{\max }$ & $\mathrm{E}_{\text {gap }}$ & $\mathrm{E}_{\text {HOMO }}$ & $\mathrm{E}_{\text {LUMO }}$ & $\mathrm{E}_{\text {gap }}$ \\
& $\mathrm{eV}$ & $/ \mathrm{eV}$ & $/ \mathrm{eV}$ & $/ \mathrm{nm}$ & $/ \mathrm{eV}$ & $/ \mathrm{eV}$ & $/ \mathrm{eV}$ & $/ \mathrm{eV}$ \\
1 & -6.00 & -3.64 & 2.56 & 525 & 2.36 & -6.04 & -3.73 & 2.31 \\
$4 \mathrm{a}$ & -5.61 & -3.23 & 2.38 & 594 & 2.09 & -5.86 & -3.76 & 2.10 \\
$4 \mathrm{~b}$ & & & & 595 & 2.08 & -5.82 & -3.76 & 2.06
\end{tabular}

${ }^{\mathrm{a}}$ Calculations were performed at the B3LYP/6-31G** level. ${ }^{\mathrm{b}}$ Optical band gaps were estimated from the wavelength of the absorption peak. ${ }^{\mathrm{c}} \mathrm{Cyclic}$ voltammetry of $\mathbf{1 a}$ and $\mathbf{4 a - c}$ were measured at a scan rate of $100 \mathrm{mV} \mathrm{s}^{-1}$ in dichloromethane solution with $\mathrm{Bu}_{4} \mathrm{NPF}_{6}(0.1 \mathrm{M})$ as supporting electrolyte, $\mathrm{Ag} / \mathrm{AgCl}$ as reference electrode and the $\mathrm{Fc} / \mathrm{Fc}^{+}$redox couple as an internal standard. HOMO and LUMO energy levels and energy gaps were calculated by the equations $\mathrm{E}_{\mathrm{HOMO}}=-\left[\mathrm{E}_{\mathrm{ox}}-\mathrm{E}_{\left(\mathrm{Fc}_{\mathrm{Fc}}+\mathrm{Fc}^{+}\right.}+\right.$ 4.8] eV, $\mathrm{E}_{\mathrm{LUMO}}=-\left[\mathrm{E}_{\mathrm{red}}-\mathrm{E}_{(\mathrm{Fc} / \mathrm{Fc}+}+4.8\right] \mathrm{eV}$ and $\mathrm{E}_{\mathrm{gap}}=\mathrm{E}_{\text {Hомо }}-\mathrm{E}_{\mathrm{LUMO}}$.

\section{Geometry and Molecular Orbitals from Calculations}
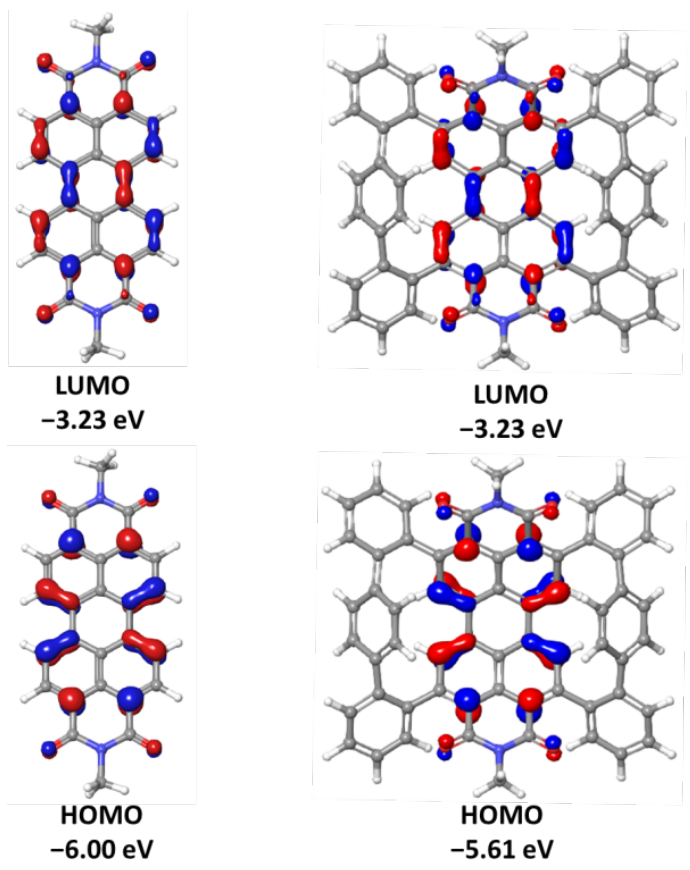

Figure S10 Lowest unoccupied molecular orbitals and highest occupied molecular orbitals for PDI core, PDI-B, all with methyl group in imides. 

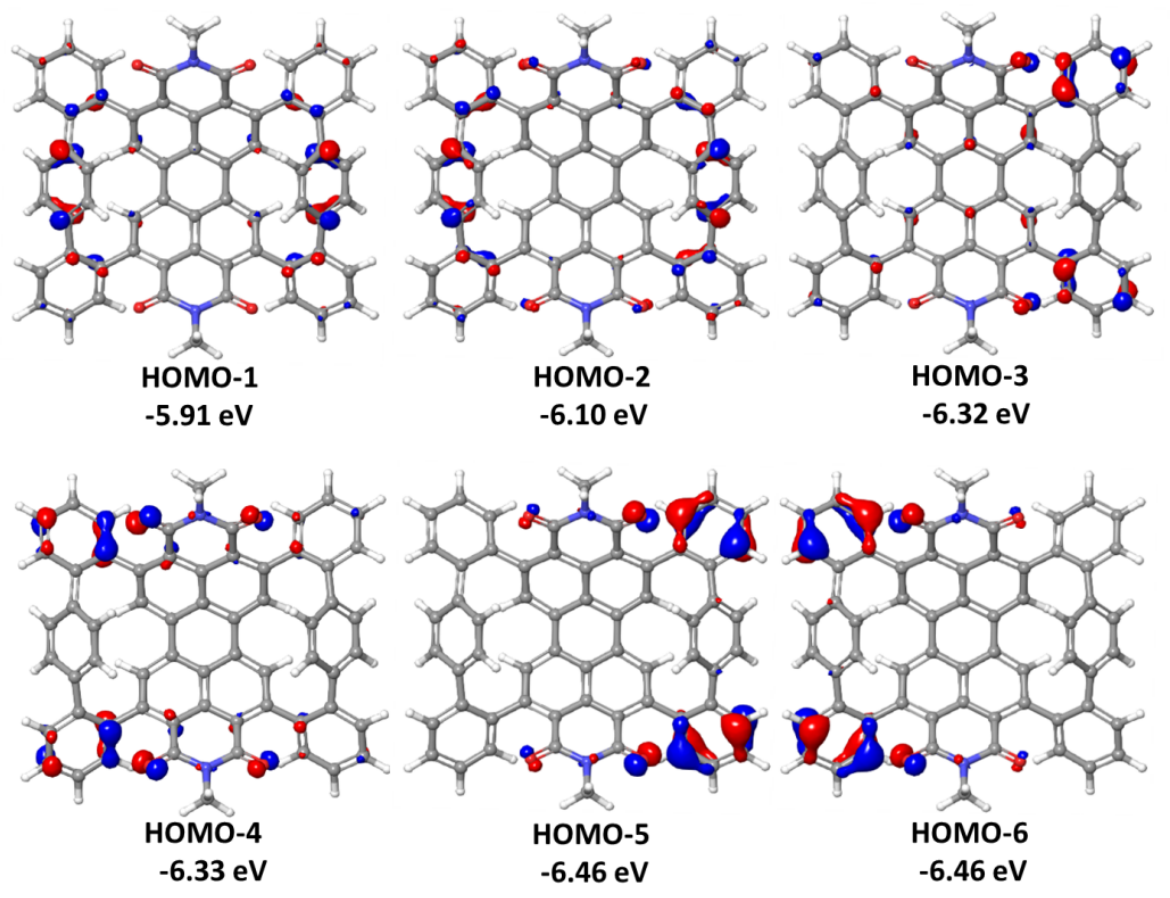

Figure S11 Other occupied orbitals obtained from calculations (B3LYP/6-31G ${ }^{* *}$ level).

\section{Sample Preparation}

Crystalline Film: A film was spin-coated on a $1 \mathrm{~cm} \times 1 \mathrm{~cm}$ sapphire substrate using 2 $\mathrm{mg}$ of PDI-B in $200 \mathrm{uL}$ of chloroform at $1000 \mathrm{rpm}$. The film was annealed using cyclohexane steam at room temperature for 2 hours.

Polymer Matrix Film: $0.5 \mathrm{~mL}$ of $1.7 \mathrm{mM}$ PMMA (M.W. $=35,000 \mathrm{~g} / \mathrm{mol}$ ) was mixed with $0.5 \mathrm{~mL}$ of $6.8 \mathrm{uM}$ PDI-B in chloroform and dropcast onto a $1 \mathrm{~cm} \mathrm{x} 1 \mathrm{~cm}$ sapphire substrate to achieve individual molecule measurements.

\section{Ultrafast Spectroscopy}

Transient Absorption (TA) Spectroscopy: A Clark-MXR IMPULSE Yb-doped fiber laser was used at a repetition rate of $800 \mathrm{kHz}$. The probe was white light generated in YAG with $250 \mathrm{fs} 1035 \mathrm{~nm}$ fundamental. The sample was pumped with $5 \mathrm{~mW}$ of second harmonic generated $515 \mathrm{~nm}$. The sample was housed inside a cryostat in a vacuum and nitrogen-cooled to $77 \mathrm{~K}$ to reduce heating affects. The TA spectrum shown in Fig. $\mathbf{2 b}$ is averaged over 20 scans of the same spot to reduce noise, showing no sample degradation over this time period. 
Time Correlated Single Photon Counting (TCSPC): Wavelength- and time-resolved photoluminescence was collected using a $514 \mathrm{~nm}$ excitation pulse (150 fs pulse duration) from a Coherent OPA 9450, pumped by a Coherent RegA 9050 Ti:Sapphire amplifier with a rep rate of $250 \mathrm{kHz}$ and pulse duration of $100 \mathrm{fs}$. The time traces were collected using a time-correlated single photon counting module (bh SPC-130). The IRF was 100 ps giving a time-resolution of $\sim 20$ ps. Wavelength-resolved TCSPC was collected from 550-900 nm, and then integrated across this spectrum for an overall decay profile. This was normalized to incident power, and optical density for each sample to obtain relative PL intensities shown in Fig. 3b. The wavelength resolved TCSPC was integrated across the time-domain to obtain the PL spectra of each sample shown in Fig. 3a.

\section{Computational Details and Crystal Data}

\section{(1) Computational Details}

Density Functional Theory (DFT) and Time Dependent DFT (TD-DFT) calculations were performed using Jaguar, version 8.2, Schrodinger, Inc., New York, NY, 2013. DFT calculations were used to estimate the exited state and triplet state of PDI core and PDIB. In order to compare the experimental spectroscopic data, we used TD-DFT to calculate the absorption spectra of PDI and PDI-B.

All quantum mechanical calculations were performed using Jaguar, version 8.2, Schrodinger, Inc., New York, NY, 2013. All geometries were optimized using the B3LYP functional and the 6-31G** basis set.

First excited singlet state energies used in the main text come from DFT HOMOLUMO calculations.

\section{PDI monomer 1 with methyl group on imide}

DFT calculation: Total energy:

-1409.76528995640 hartrees

HOMO energy: $\quad-0.22061$ hartrees $(-6.00 \mathrm{eV})$

LUMO energy: $\quad-0.12747$ hartrees $(-3.47 \mathrm{eV})$

Final geometry:

\begin{tabular}{lccc} 
& \multicolumn{3}{c}{ angstroms } \\
atom & $\mathrm{x}$ & $\mathrm{y}$ & $\mathrm{Z}$ \\
$\mathrm{N} 1$ & -0.0284099543 & -0.0004755076 & -0.0275605898 \\
$\mathrm{C} 2$ & 0.0085487954 & 0.0006714215 & 1.3722104732 \\
$\mathrm{C} 3$ & 1.3474774345 & 0.0014561197 & 2.0100302654 \\
$\mathrm{C} 4$ & 2.5243416286 & 0.0012325967 & 1.2232767808 \\
$\mathrm{C} 5$ & 2.4214167829 & 0.0006007630 & -0.1888877692
\end{tabular}




\begin{tabular}{|c|c|c|c|}
\hline C6 & 1.0990049743 & 0.0004072291 & -0.8628747867 \\
\hline $\mathrm{C} 7$ & 3.5706780416 & 0.0010205053 & -0.9596812054 \\
\hline $\mathrm{C} 8$ & 4.8307435349 & 0.0003778635 & -0.3534880315 \\
\hline C9 & 4.9833118002 & -0.0006031434 & 1.0347012164 \\
\hline $\mathrm{C} 10$ & 3.8086816190 & 0.0016457164 & 1.8515074450 \\
\hline $\mathrm{C} 11$ & 3.8862701731 & 0.0041632132 & 3.2804579699 \\
\hline $\mathrm{C} 12$ & 5.2086105233 & 0.0078578729 & 3.9254607989 \\
\hline C13 & 6.3832081823 & 0.0017263284 & 3.1085311575 \\
\hline $\mathrm{C} 14$ & 6.3049026102 & -0.0041707580 & 1.6798567369 \\
\hline $\mathrm{C} 15$ & 7.4942062311 & -0.0131163830 & 0.9473516760 \\
\hline $\mathrm{C} 16$ & 8.7473077568 & -0.0145046575 & 1.5685570088 \\
\hline C17 & 8.8450066505 & -0.0067313310 & 2.9485589661 \\
\hline C18 & 7.6682945239 & 0.0014404237 & 3.7356975136 \\
\hline C19 & 5.3630598948 & 0.0169448722 & 5.3132612261 \\
\hline $\mathrm{C} 20$ & 6.6235172978 & 0.0177593345 & 5.9188098170 \\
\hline $\mathrm{C} 21$ & 7.7724361465 & 0.0093272411 & 5.1476445649 \\
\hline $\mathrm{C} 22$ & 9.0954356312 & 0.0092643744 & 5.8208137024 \\
\hline $\mathrm{N} 23$ & 10.2224096448 & 0.0006675862 & 4.9849126215 \\
\hline $\mathrm{C} 24$ & 10.1843436915 & -0.0075539588 & 3.5852971754 \\
\hline $\mathrm{C} 25$ & 1.4443408660 & 0.0017907645 & 3.3904007120 \\
\hline $\mathrm{C} 26$ & 2.6970056543 & 0.0030151381 & 4.0132650653 \\
\hline $\mathrm{O} 27$ & 11.2247892853 & -0.0149596543 & 2.9407181854 \\
\hline $\mathrm{O} 28$ & 9.2067413395 & 0.0163026860 & 7.0396196920 \\
\hline $\mathrm{O} 29$ & -1.0325536240 & 0.0008267559 & 2.0158066894 \\
\hline $\mathrm{O} 30$ & 0.9885089485 & 0.0006796677 & -2.0818384494 \\
\hline H31 & 7.4665554049 & -0.0198292276 & -0.1352542274 \\
\hline H32 & 9.6603562913 & -0.0213738294 & 0.9833826217 \\
\hline H33 & 6.7232516002 & 0.0247215688 & 6.9986039581 \\
\hline H34 & 4.4930583010 & 0.0242153798 & 5.9575505989 \\
\hline $\mathrm{C} 35$ & 11.5541929938 & -0.0003334077 & 5.5989422152 \\
\hline H36 & 0.5306597336 & 0.0011258102 & 3.9746309083 \\
\hline H37 & 2.7245822395 & 0.0027294893 & 5.0958407829 \\
\hline H38 & 5.7019022386 & 0.0009605217 & -0.9968434294 \\
\hline H39 & 3.4713180001 & 0.0015498158 & -2.0395437669 \\
\hline $\mathrm{C} 40$ & -1.3596129030 & -0.0008459825 & -0.6428051875 \\
\hline H41 & 11.4211379366 & 0.0066032246 & 6.6778341146 \\
\hline H42 & 12.1053627927 & -0.8889419063 & 5.2843981146 \\
\hline $\mathrm{H} 43$ & 12.1115927172 & 0.8805279864 & 5.2737865507 \\
\hline H44 & -1.2254322746 & -0.0023427007 & -1.7215855584 \\
\hline $\mathrm{H} 45$ & -1.9144275180 & -0.8850130665 & -0.3223010162 \\
\hline H46 & -1.9139479445 & 0.8845508191 & -0.3248128828 \\
\hline
\end{tabular}

TD-DFT calculations: 
Total energy: -1409.67555374 hartrees

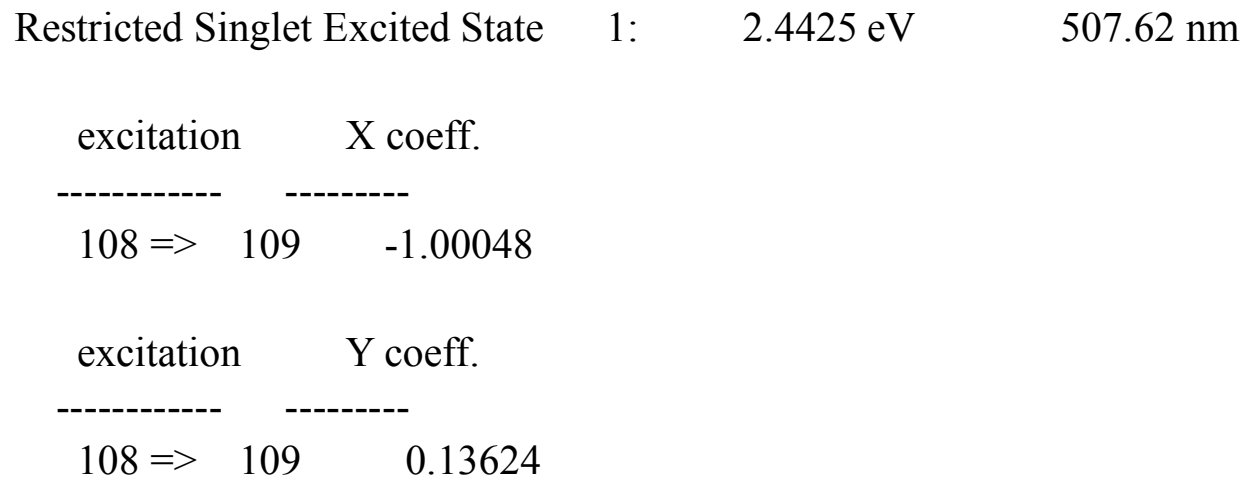

Transition dipole moment (debye):

$\begin{aligned} \mathrm{X}= & -7.5293 \quad \mathrm{Y}=\quad 0.0046 \quad \mathrm{Z}=-3.9275 \quad \mathrm{Tot}= \\ 8.4920 & \end{aligned}$

Oscillator strength, $\mathrm{f}=\quad 0.6680$

Restricted Singlet Excited State $\quad 2: \quad 3.1457 \mathrm{eV} \quad 394.14 \mathrm{~nm}$

\begin{tabular}{ccc} 
excitation & \multicolumn{2}{c}{ X coeff. } \\
\hdashline $106=>$ & 110 & 0.18716 \\
$107=>$ & 109 & 0.97511
\end{tabular}

Transition dipole moment (debye):
$\mathrm{X}=$
0.0007
$\mathrm{Y}=$
0.1048
$\mathrm{Z}=$
0.0000 Tot $=$

0.1048

Oscillator strength, $\mathrm{f}=\quad 0.0001$

Restricted Singlet Excited State 3: $\quad 3.1504 \mathrm{eV} \quad 393.55 \mathrm{~nm}$

\begin{tabular}{|c|c|c|}
\hline excitation & & $X$ coeff. \\
\hline & & \\
\hline $107=>$ & 110 & -0.18832 \\
\hline
\end{tabular}

Transition dipole moment (debye):

$\begin{array}{lll}\mathrm{X}= & -0.0004 & \mathrm{Y}= \\ 0.0066\end{array}$ 
Oscillator strength, $\mathrm{f}=$ 0.0000

Restricted Singlet Excited State $\quad 4: \quad 3.3788 \mathrm{eV} \quad 366.95 \mathrm{~nm}$

\begin{tabular}{ccc} 
excitation & \multicolumn{2}{c}{ X coeff. } \\
--------- & -------- \\
100 & $\Rightarrow 109$ & -0.10394 \\
103 & $\Rightarrow 109$ & -0.70179 \\
105 & $\Rightarrow 109$ & 0.64741 \\
108 & $\Rightarrow 112$ & -0.25453
\end{tabular}

Transition dipole moment (debye):

$$
\mathrm{X}=-0.0005 \quad \mathrm{Y}=-0.0043 \quad \mathrm{Z}=-0.0036 \quad \mathrm{Tot}=
$$

0.0056

Oscillator strength, $\mathrm{f}=\quad 0.0000$

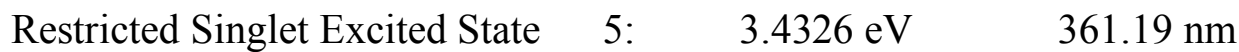

\begin{tabular}{ccc} 
excitation & \multicolumn{2}{c}{ X coeff. } \\
\hdashline------ & ---- \\
100 & $\Rightarrow 109$ & 0.25897 \\
103 & $\Rightarrow 109$ & 0.64690 \\
105 & $\Rightarrow 109$ & 0.70176 \\
108 & $\Rightarrow>112$ & -0.10376
\end{tabular}

Transition dipole moment (debye):

$$
\mathrm{X}=-0.0014 \quad \mathrm{Y}=-0.0004 \quad \mathrm{Z}=-0.0033 \quad \mathrm{Tot}=
$$

0.0036

Oscillator strength, $\mathrm{f}=\quad 0.0000$

\begin{tabular}{|c|c|c|}
\hline excitatio & & coeff. \\
\hline & & \\
\hline $101=$ & 109 & -0.17264 \\
\hline $104=>$ & 109 & 0.96102 \\
\hline $108=>$ & 113 & 0.15655 \\
\hline
\end{tabular}

$\begin{array}{lll}\text { Restricted Singlet Excited State } \quad 6: \quad 3.5162 \mathrm{eV} & 352.60 \mathrm{~nm}\end{array}$

Transition dipole moment (debye): 


$\mathrm{X}=$
0.4481

Oscillator strength, $\mathrm{f}=\quad 0.0027$

Restricted Singlet Excited State 7: $\quad 3.5535 \mathrm{eV} \quad 348.91 \mathrm{~nm}$

\begin{tabular}{ccc} 
excitation & \multicolumn{1}{c}{ X coeff. } \\
-------- & --- \\
$102 \Rightarrow$ & 109 & -0.65608 \\
$108 \Rightarrow$ & 110 & -0.74931
\end{tabular}

Transition dipole moment (debye):

$\begin{array}{cc}\mathrm{X}= & -0.0006 \quad \mathrm{Y}=-0.0002 \quad \mathrm{Z}=0.0003 \quad \mathrm{Tot}= \\ 0.0007 & \end{array}$

Oscillator strength, $\mathrm{f}=\quad 0.0000$

Restricted Singlet Excited State $\quad 8: \quad 3.6563 \mathrm{eV} \quad 339.10 \mathrm{~nm}$

\begin{tabular}{|c|c|c|}
\hline excitatio & & coeff. \\
\hline 100 & 100 & -041244 \\
\hline $103=>$ & 109 & 0.12214 \\
\hline $108=>$ & 111 & 0.89663 \\
\hline
\end{tabular}

Transition dipole moment (debye):

$$
\mathrm{X}=0.0048 \quad \mathrm{Y}=0.0003 \quad \mathrm{Z}=0.0034 \text { Tot }=
$$
0.0059

Oscillator strength, $\mathrm{f}=\quad 0.0000$

Restricted Singlet Excited State 9: $\quad 3.6664 \mathrm{eV} \quad 338.16 \mathrm{~nm}$

\begin{tabular}{rrr} 
excitation & \multicolumn{2}{c}{ X coeff. } \\
-------- & --18618 \\
98 & $=>110$ & -0.186 \\
107 & $\Rightarrow 109$ & -0.96861 \\
& $>111$ & 0.11612
\end{tabular}

Transition dipole moment (debye): 


$\begin{aligned} & \mathrm{X}=-0.0049 \\ & 0.0053\end{aligned}$

Oscillator strength, $\mathrm{f}=\quad 0.0000$

Restricted Singlet Excited State 10 : $\quad 3.6778 \mathrm{eV} \quad 337.12 \mathrm{~nm}$

\begin{tabular}{rrr} 
excitation & \multicolumn{2}{c}{ X coeff. } \\
-98 & $=>109$ & -0.96736 \\
99 & $\Rightarrow 110$ & -0.18914 \\
106 & $\Rightarrow 111$ & 0.11971
\end{tabular}

Transition dipole moment (debye):

$$
\mathrm{X}=-0.0040 \quad \mathrm{Y}=-0.0005 \quad \mathrm{Z}=0.0076 \quad \text { Tot }=
$$

0.0085

Oscillator strength, $\mathrm{f}=\quad 0.0000$

$\begin{array}{lll}\text { Restricted Singlet Excited State } & 11: & 3.7728 \mathrm{eV}\end{array} 328.63 \mathrm{~nm}$

\begin{tabular}{|c|c|c|}
\hline excitation & \multicolumn{2}{|c|}{$\mathrm{X}$ coeff. } \\
\hline & & \\
\hline $101=>$ & 109 & -0.91349 \\
\hline $102=>$ & 112 & -0.11712 \\
\hline $104 \Rightarrow$ & 109 & -0.22122 \\
\hline $108=>$ & 113 & 0.31320 \\
\hline
\end{tabular}

Transition dipole moment (debye):

2.3893
$\mathrm{X}=$
1.1072
$\mathrm{Y}=$
$-0.0065$
$\mathrm{Z}=$
-2.1173 Tot $=$

Oscillator strength, $\mathrm{f}=$

0.0817

$\begin{array}{lll}\text { Restricted Singlet Excited State } & 12: & 3.8427 \mathrm{eV}\end{array} 322.65 \mathrm{~nm}$

\begin{tabular}{ccc} 
excitation & \multicolumn{2}{c}{ X coeff. } \\
--------- & ------ \\
100 & $=>109$ & 0.72081 \\
103 & $=>109$ & -0.12132 \\
105 & $=>109$ & -0.23327
\end{tabular}




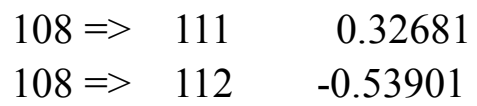

Transition dipole moment (debye):

$$
\mathrm{X}=0.0039 \quad \mathrm{Y}=-0.0042 \quad \mathrm{Z}=-0.0045 \text { Tot }=
$$

0.0073

Oscillator strength, $\mathrm{f}=\quad 0.0000$

$\begin{array}{lll}\text { Restricted Singlet Excited State } & 13: & 3.9290 \mathrm{eV}\end{array} 315.56 \mathrm{~nm}$

\begin{tabular}{|c|c|c|}
\hline excitatior & & coeff. \\
\hline & & \\
\hline $102=$ & 109 & -0.73475 \\
\hline $108=>$ & 110 & 0.64103 \\
\hline
\end{tabular}

Transition dipole moment (debye):

$$
\mathrm{X}=-0.0020 \quad \mathrm{Y}=\quad 0.0013 \quad \mathrm{Z}=-0.0010 \quad \text { Tot }=
$$

0.0025

Oscillator strength, $\mathrm{f}=\quad 0.0000$

\begin{tabular}{|c|c|c|}
\hline excitat & & coeff. \\
\hline & & \\
\hline $100=>$ & 109 & 5977 \\
\hline $101=>$ & 110 & 0.18488 \\
\hline $103 \Rightarrow$ & 109 & 0.21769 \\
\hline $105=>$ & 109 & -0.13763 \\
\hline $108 \Rightarrow$ & 111 & -0.26681 \\
\hline $108=>$ & 112 & -0.77369 \\
\hline
\end{tabular}

Restricted Singlet Excited State 14: $\quad 4.2935 \mathrm{eV} \quad 288.77 \mathrm{~nm}$

Transition dipole moment (debye):

$$
\mathrm{X}=0.0053 \quad \mathrm{Y}=\quad 0.0150 \quad \mathrm{Z}=-0.0038 \text { Tot }=
$$
0.0164

Oscillator strength, $\mathrm{f}=\quad 0.0000$

$\begin{array}{lll}\text { Restricted Singlet Excited State } & 15: \quad 4.7161 \mathrm{eV} & 262.89 \mathrm{~nm}\end{array}$ 


\begin{tabular}{rlr} 
excitation & \multicolumn{1}{c}{ X coeff. } \\
--------- & -------- \\
98 & $\Rightarrow 111$ & -0.14900 \\
99 & $\Rightarrow 115$ & -0.10127 \\
106 & $\Rightarrow 109$ & -0.21575 \\
106 & $\Rightarrow 114$ & -0.21239 \\
107 & $\Rightarrow 110$ & 0.92528
\end{tabular}

Transition dipole moment (debye):

$$
\mathrm{X}=-0.0015 \quad \mathrm{Y}=0.0006 \quad \mathrm{Z}=0.0045 \text { Tot }=
$$
0.0048

Oscillator strength, $\mathrm{f}=\quad 0.0000$

Triplet calculation:

Total energy: $\quad-1409.71698399908$ hartrees

final geometry:

angstroms

$\begin{array}{lccc}\text { atom } & \mathrm{x} & \mathrm{y} & \mathrm{z} \\ \mathrm{N} 1 & -0.0093847945 & 0.0003452956 & -0.0188638295 \\ \mathrm{C} 2 & 0.0286443898 & 0.0036310599 & 1.3799488056 \\ \mathrm{C} 3 & 1.3608017844 & 0.0034216896 & 2.0142741120 \\ \mathrm{C} 4 & 2.5358992197 & 0.0005383254 & 1.2307394140 \\ \mathrm{C} 5 & 2.4328806059 & -0.0023628857 & -0.1779575997 \\ \mathrm{C} 6 & 1.1189843836 & -0.0020328605 & -0.8507967779 \\ \mathrm{C} 7 & 3.6001327071 & -0.0043568821 & -0.9660687353 \\ \mathrm{C} 8 & 4.8397723660 & -0.0044999219 & -0.3754248278 \\ \mathrm{C} 9 & 4.9997059077 & -0.0026593673 & 1.0494336001 \\ \mathrm{C} 10 & 3.8249065327 & 0.0006820486 & 1.8610547820 \\ \mathrm{C} 11 & 3.9050659855 & 0.0042343773 & 3.2866266407 \\ \mathrm{C} 12 & 5.1917822301 & 0.0058814262 & 3.9136972068 \\ \mathrm{C} 13 & 6.3664174258 & 0.0010818870 & 3.1013100951 \\ \mathrm{C} 14 & 6.2853964117 & -0.0038632123 & 1.6762650081 \\ \mathrm{C} 15 & 7.5077082048 & -0.0099632838 & 0.9245186309 \\ \mathrm{C} 16 & 8.7367528797 & -0.0102851622 & 1.5382567457 \\ \mathrm{C} 17 & 8.8306830437 & -0.0042802962 & 2.9434423824 \\ \mathrm{C} 18 & 7.6565722450 & 0.0012561688 & 3.7292417309 \\ \mathrm{C} 19 & 5.3542917797 & 0.0124370881 & 5.3385640812 \\ \mathrm{C} 20 & 6.5951909191 & 0.0130391615 & 5.9272722194\end{array}$




$\begin{array}{lrcc}\text { C21 } & 7.7616649046 & 0.0069439240 & 5.1376404112 \\ \text { C22 } & 9.0768810732 & 0.0073329738 & 5.8080446100 \\ \text { N23 } & 10.2038314508 & 0.0015722780 & 4.9739127118 \\ \text { C24 } & 10.1635230860 & -0.0043661191 & 3.5749180794 \\ \text { C25 } & 1.4531344323 & 0.0056477028 & 3.4201475098 \\ \text { C26 } & 2.6811395568 & 0.0059433552 & 4.0370214110 \\ \text { O27 } & 11.2048295961 & -0.0092872676 & 2.9248218354 \\ \text { O28 } & 9.1847331964 & 0.0123966633 & 7.0308946943 \\ \text { O29 } & -1.0137278265 & 0.0064987297 & 2.0283730716 \\ \text { O30 } & 1.0138552748 & -0.0038606271 & -2.0739212340 \\ \text { H31 } & 7.4719869965 & -0.0154354051 & -0.1572797860 \\ \text { H32 } & 9.6558879123 & -0.0150607537 & 0.9636648230 \\ \text { H33 } & 6.7076027295 & 0.0183125204 & 7.0054191810 \\ \text { H34 } & 4.4810791712 & 0.0182315413 & 5.9776021754 \\ \text { C35 } & 11.5347299832 & 0.0013797870 & 5.5890017218 \\ \text { H36 } & 0.5329095181 & 0.0068856561 & 3.9930594650 \\ \text { H37 } & 2.7146107281 & 0.0068010120 & 5.1187979660 \\ \text { H38 } & 5.7142953153 & -0.0051714895 & -1.0127380413 \\ \text { H39 } & 3.4892672557 & -0.0055634252 & -2.0443682032 \\ \text { C40 } & -1.3392956547 & 0.0014536061 & -0.6358101786 \\ \text { H41 } & 11.3999937836 & 0.0063563394 & 6.6677862640 \\ \text { H42 } & 12.0872920646 & -0.8860337492 & 5.2735913334 \\ \text { H43 } & 12.0911148877 & 0.8836096735 & 5.2658783758 \\ \text { H44 } & -1.2029334302 & -0.0018628581 & -1.7143739125 \\ \text { H45 } & -1.8960043308 & -0.8812890657 & -0.3146827241 \\ \text { H46 } & -1.8922709393 & 0.8884103662 & -0.3197984064\end{array}$

\section{PDI-based Bowl 4 with methyl group on imide}

DFT calculation:

Total energy: $\quad-2793.62518049211$ hartrees

HOMO energy: $\quad-0.20617$ hartrees $\quad(-5.61 \mathrm{eV})$

LUMO energy: $\quad-0.11871$ hartrees $(-3.23 \mathrm{eV})$

Final geometry:

$\begin{array}{lccc}\mathrm{N} 1 & 0.1186831061 & -0.0090652407 & -0.0398597068 \\ \mathrm{C} 2 & 0.0966652261 & -0.1663760168 & 1.3529133379 \\ \mathrm{C} 3 & 1.4079873662 & -0.1147873636 & 2.0520112079 \\ \mathrm{C} 4 & 2.5533942499 & 0.3355969174 & 1.3234399471 \\ \mathrm{C} 5 & 2.5311070953 & 0.4679088920 & -0.1003883858\end{array}$




\begin{tabular}{|c|c|c|c|}
\hline C6 & 1.2131015282 & 0.4431309514 & -0.7915368186 \\
\hline $\mathrm{C} 7$ & 3.7112505150 & 0.7540034882 & 2.0409024537 \\
\hline $\mathrm{C} 8$ & 3.7105540460 & 0.7186616733 & 3.4637145053 \\
\hline C9 & 2.6918399688 & 0.0209454902 & 4.0991013514 \\
\hline $\mathrm{C} 10$ & 1.5140523285 & -0.3741784395 & 3.4325818067 \\
\hline $\mathrm{C} 11$ & 4.8360647436 & 1.2626477092 & 1.3341743117 \\
\hline $\mathrm{C} 12$ & 5.7664945321 & 2.1429296235 & 2.0539361637 \\
\hline C13 & 5.4868496511 & 2.4491315449 & 3.4153144107 \\
\hline $\mathrm{C} 14$ & 4.6311140709 & 1.6108618000 & 4.1816356301 \\
\hline $\mathrm{C} 15$ & 3.7247476950 & 0.7478439486 & -0.7959841449 \\
\hline $\mathrm{C} 16$ & 4.8687887673 & 1.0970904135 & -0.0464587099 \\
\hline C17 & 6.0175876311 & 3.6365825884 & 3.9963515122 \\
\hline C18 & 6.8194557406 & 4.5053151666 & 3.1933001867 \\
\hline C19 & 7.2823393573 & 4.0684612748 & 1.9375979936 \\
\hline $\mathrm{C} 20$ & 6.7641875872 & 2.8664623817 & 1.4147571176 \\
\hline $\mathrm{C} 21$ & 5.6601547644 & 3.9768944125 & 5.3379963949 \\
\hline $\mathrm{C} 22$ & 5.8641095842 & 5.3790149618 & 5.7908314912 \\
\hline $\mathrm{N} 23$ & 6.6480939312 & 6.1986598859 & 4.9667582679 \\
\hline $\mathrm{C} 24$ & 7.0231090422 & 5.9053338190 & 3.6482600843 \\
\hline $\mathrm{C} 25$ & 5.0112836231 & 3.0265771689 & 6.1513896525 \\
\hline $\mathrm{C} 26$ & 4.5467979619 & 1.8381794489 & 5.5501366586 \\
\hline $\mathrm{C} 27$ & 0.4401941752 & -1.0488959995 & 4.2291221894 \\
\hline $\mathrm{C} 28$ & -0.0763720846 & -2.2562944510 & 3.7310720386 \\
\hline $\mathrm{C} 29$ & -1.0507925350 & -2.9762382106 & 4.4063130714 \\
\hline $\mathrm{C} 30$ & -1.5487006598 & -2.4900770487 & 5.6143982246 \\
\hline C31 & -1.0253351906 & -1.3179275714 & 6.1458830538 \\
\hline $\mathrm{C} 32$ & -0.0169027559 & -0.5881365384 & 5.4919400355 \\
\hline $\mathrm{C} 33$ & 3.8314849207 & 0.6900993327 & -2.2905642722 \\
\hline C34 & 4.4638261287 & 1.6869011188 & -3.0805230778 \\
\hline $\mathrm{C} 35$ & 4.4875627574 & 1.5187523683 & -4.4764747719 \\
\hline $\mathrm{C} 36$ & 3.9514389901 & 0.3950270979 & -5.0956033260 \\
\hline $\mathrm{C} 37$ & 3.3934367105 & -0.6156519115 & -4.3127918162 \\
\hline $\mathrm{C} 38$ & 3.3372279930 & -0.4570471623 & -2.9350289714 \\
\hline C39 & 8.2962478200 & 4.8208526873 & 1.1329361449 \\
\hline $\mathrm{C} 40$ & 9.4760916919 & 5.2206060482 & 1.7841363691 \\
\hline $\mathrm{C} 41$ & 10.4847976926 & 5.9054323914 & 1.1218586074 \\
\hline $\mathrm{C} 42$ & 10.3298848528 & 6.2190039012 & -0.2280131736 \\
\hline $\mathrm{C} 43$ & 9.1894756454 & 5.7989905795 & -0.9009713912 \\
\hline $\mathrm{C} 44$ & 8.1670319162 & 5.0815385002 & -0.2566082467 \\
\hline $\mathrm{C} 45$ & 4.7950623314 & 3.2163387212 & 7.6211167878 \\
\hline $\mathrm{C} 46$ & 3.5673021130 & 2.9515639778 & 8.2830469058 \\
\hline $\mathrm{C} 47$ & 3.4923701953 & 3.1761933322 & 9.6684921395 \\
\hline $\mathrm{C} 48$ & 4.5896484926 & 3.6024845147 & 10.4066020281 \\
\hline C49 & 5.8130736372 & 3.7935929389 & 9.7661115021 \\
\hline
\end{tabular}




\begin{tabular}{|c|c|c|c|}
\hline $\mathrm{C} 50$ & 5.9012500656 & 3.6049485364 & 8.3949317515 \\
\hline $\mathrm{O} 51$ & -0.9803258785 & -0.2851550810 & 1.9216600937 \\
\hline $\mathrm{O} 52$ & 1.0329443089 & 0.8323751296 & -1.9374030710 \\
\hline $\mathrm{O} 53$ & 7.4440026590 & 6.8051073479 & 2.9337821595 \\
\hline O54 & 5.3542394171 & 5.8542082077 & 6.7961719107 \\
\hline C55 & 0.6356544532 & 0.5151863080 & 6.2613072221 \\
\hline $\mathrm{C} 56$ & 1.1663943867 & 0.1741680993 & 7.5154092010 \\
\hline $\mathrm{C} 57$ & 2.0304310699 & 1.0302049701 & 8.1885430553 \\
\hline $\mathrm{C} 58$ & 2.4044936116 & 2.2656903821 & 7.6382932298 \\
\hline C59 & 1.7565674342 & 2.6729862588 & 6.4610257784 \\
\hline C60 & 0.8863973680 & 1.8129416859 & 5.7846081979 \\
\hline C61 & 7.0981915518 & 4.4879947748 & -1.1174087846 \\
\hline C62 & 7.5242012567 & 3.6608487249 & -2.1680867263 \\
\hline C63 & 6.6295147288 & 2.8429422763 & -2.8496444826 \\
\hline C64 & 5.2678827392 & 2.8138065791 & -2.5110335334 \\
\hline C65 & 4.8163830340 & 3.7505094007 & -1.5670293325 \\
\hline C66 & 5.7155493810 & 4.5738345725 & -0.8833885818 \\
\hline C67 & 6.8364463852 & 7.5850242227 & 5.4076924501 \\
\hline C68 & -1.1819577690 & -0.0336962088 & -0.7179996749 \\
\hline H69 & 2.7468218813 & -0.1462509737 & 5.1639591525 \\
\hline $\mathrm{H} 70$ & 5.7702831857 & 1.3384033198 & -0.5901880035 \\
\hline H71 & 7.0966457384 & 2.5606809545 & 0.4346153749 \\
\hline H72 & 4.0256077085 & 1.1289173137 & 6.1759679567 \\
\hline H73 & 0.3076310048 & -2.6349149772 & 2.7895928726 \\
\hline H74 & 9.5968089857 & 4.9788429233 & 2.8350761257 \\
\hline $\mathrm{H} 75$ & -1.4229016689 & -3.9073082512 & 3.9897911744 \\
\hline H76 & 11.3832355982 & 6.1977719578 & 1.6571137146 \\
\hline $\mathrm{H} 77$ & -2.3261478631 & -3.0286894712 & 6.1483729370 \\
\hline $\mathrm{H} 78$ & 11.0996277699 & 6.7727958491 & -0.7574915731 \\
\hline H79 & -1.3912564283 & -0.9496957848 & 7.0999529529 \\
\hline H80 & 9.0766467482 & 6.0199538656 & -1.9582121321 \\
\hline H81 & 0.9743170334 & -0.8148286814 & 7.9205669249 \\
\hline H82 & 8.5849797959 & 3.5884188021 & -2.3885947156 \\
\hline H83 & 2.4998449234 & 0.6971354043 & 9.1091982469 \\
\hline H84 & 7.0054489537 & 2.1451680891 & -3.5919256550 \\
\hline H85 & 1.9786233966 & 3.6482814668 & 6.0384300117 \\
\hline H86 & 3.7592507447 & 3.7974177793 & -1.3232884242 \\
\hline H87 & 0.4430010779 & 2.1326315130 & 4.8462894937 \\
\hline H88 & 5.3436586973 & 5.2494582474 & -0.1190301168 \\
\hline H89 & 2.5451950737 & 2.9968899881 & 10.1685334943 \\
\hline H90 & 4.9494808730 & 2.2949996622 & -5.0795780912 \\
\hline H91 & 4.4948369763 & 3.7659219258 & 11.4761454558 \\
\hline H92 & 3.9866618640 & 0.3016822505 & -6.1769303463 \\
\hline H93 & 6.6915999428 & 4.0934344713 & 10.3294923562 \\
\hline
\end{tabular}




$\begin{array}{lrrr}\text { H94 } & 2.9995263805 & -1.5175613517 & -4.7710942631 \\ \text { H95 } & 6.8536917886 & 3.7579192349 & 7.8979666359 \\ \text { H96 } & 2.9000656263 & -1.2434190986 & -2.3282193380 \\ \text { H97 } & -1.0152510221 & -0.2714975534 & -1.7660629816 \\ \text { H98 } & -1.8074531301 & -0.7799004607 & -0.2322681439 \\ \text { H99 } & -1.6793954686 & 0.9387208982 & -0.6497922761 \\ \text { H100 } & 6.8959248613 & 7.5928177749 & 6.4937726985 \\ \text { H101 } & 7.7483475986 & 7.9674353807 & 4.9539543422 \\ \text { H102 } & 5.9974785749 & 8.2146326849 & 5.0955791249\end{array}$

TD-DFT calculations:

Total energy: $\quad-2793.54776201$ hartrees

Restricted Singlet Excited State 1:

Excitation energy $=\quad 0.0774290127$ hartrees $\quad 2.10695064 \mathrm{eV}$ $588.45 \mathrm{~nm}$

excitation $\mathrm{X}$ coeff.

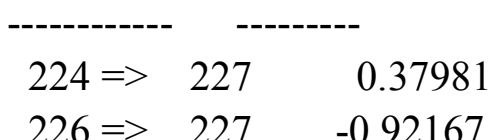

Transition dipole moment (debye):

$\mathrm{X}=-3.6770 \quad \mathrm{Y}=-3.0832 \quad \mathrm{Z}=-2.0971 \quad \mathrm{Tot}=$ 5.2368

Oscillator strength, $\mathrm{f}=\quad 0.2191$

Restricted Singlet Excited State $\quad 2$ :

Excitation energy $=\quad 0.0794856674$ hartrees $\quad 2.16291506 \mathrm{eV}$ $573.23 \mathrm{~nm}$

excitation $\mathrm{X}$ coeff.

$225=>227 \quad-0.99385$

Transition dipole moment (debye):

$\mathrm{X}=0.1166 \quad \mathrm{Y}=0.0473 \quad \mathrm{Z}=0.0013 \quad \mathrm{Tot}=$ 0.1259

Oscillator strength, $\mathrm{f}=\quad 0.0001$

Restricted Singlet Excited State 3:

Excitation energy $=0.0885633595$ hartrees $\quad 2.40993163 \mathrm{eV}$ $514.47 \mathrm{~nm}$ 


\begin{tabular}{|c|c|c|}
\hline \multicolumn{2}{|c|}{ excitation } & $X$ coeff. \\
\hline & & \\
\hline $220=>$ & 227 & -0.13017 \\
\hline $221=>$ & 227 & 0.15350 \\
\hline $224=>$ & 227 & 0.89963 \\
\hline $226=>$ & 227 & 0.37173 \\
\hline
\end{tabular}

Transition dipole moment (debye):

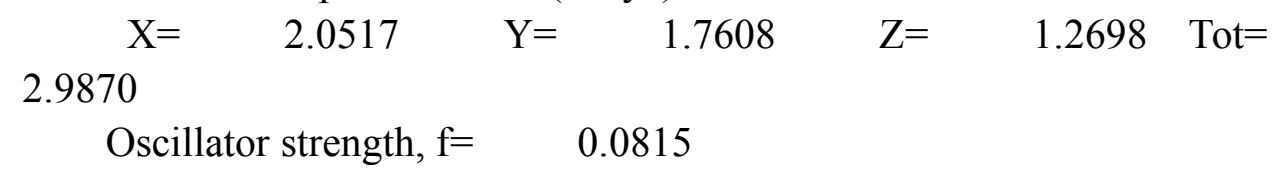

Restricted Singlet Excited State $\quad 4$ :

Excitation energy $=0.0942578997$ hartrees $\quad 2.56488795 \mathrm{eV}$ $483.39 \mathrm{~nm}$

excitation $\mathrm{X}$ coeff.

$$
\begin{aligned}
& 216=>227 \quad-0.15379 \\
& 222 \Rightarrow 227 \quad-0.94550 \\
& 223=>227 \quad-0.23013
\end{aligned}
$$

Transition dipole moment (debye):

$$
\begin{aligned}
& \mathrm{X}=0.2402 \quad \mathrm{Y}=\quad-0.2567 \quad \mathrm{Z}=0.2998 \quad \mathrm{Tot}= \\
& 0.4620
\end{aligned}
$$

\begin{tabular}{|c|c|c|}
\hline excita & tion & X coeff. \\
\hline 222 & 027 & 160 \\
\hline $223=>$ & 227 & 0.95507 \\
\hline
\end{tabular}

Restricted Singlet Excited State 5:

Excitation energy $=0.0958514306$ hartrees $\quad 2.60825013 \mathrm{eV}$ $475.35 \mathrm{~nm}$

Transition dipole moment (debye):

$$
\mathrm{X}=1.8141 \quad \mathrm{Y}=0.3072 \quad \mathrm{Z}=-3.5219 \text { Tot }=
$$

3.9735

Oscillator strength, $\mathrm{f}=\quad 0.1562$

Restricted Singlet Excited State $\quad 6$ :

Excitation energy $=0.0988256400$ hartrees $\quad 2.68918249 \mathrm{eV}$ $461.05 \mathrm{~nm}$

excitation $\mathrm{X}$ coeff. 


\begin{tabular}{|c|c|c|}
\hline $213=>$ & 227 & -0.11876 \\
\hline $220=>$ & 227 & 0.60063 \\
\hline $221=>$ & 227 & -0.74564 \\
\hline $224=>$ & 227 & 0.19442 \\
\hline
\end{tabular}

Transition dipole moment (debye):

$\mathrm{X}=1.7231 \quad \mathrm{Y}=1.4698 \quad \mathrm{Z}=1.0140 \quad \mathrm{Tot}=$ 2.4814

Oscillator strength, $\mathrm{f}=\quad 0.0628$

Restricted Singlet Excited State 7:

Excitation energy $=0.1006817457$ hartrees $\quad 2.73968970 \mathrm{eV}$ $452.55 \mathrm{~nm}$

excitation $\quad \mathrm{X}$ coeff.

$220=227 \quad 0.77021$

$221=227 \quad 0.62256$

Transition dipole moment (debye):

$\mathrm{X}=0.0043 \quad \mathrm{Y}=0.0192 \quad \mathrm{Z}=0.1344$ Tot $=$ 0.1359

Oscillator strength, $\mathrm{f}=\quad 0.0002$

Restricted Singlet Excited State $\quad 8$ :

Excitation energy $=0.1111924071$ hartrees $\quad 3.02569934 \mathrm{eV}$ $409.77 \mathrm{~nm}$

\begin{tabular}{|c|c|}
\hline excitati & X coeff. \\
\hline $219=>$ & -0.987 \\
\hline
\end{tabular}

Transition dipole moment (debye):
$\mathrm{X}=$
1.0948
$\mathrm{Y}=$
0.1993
$\mathrm{Z}=$
-2.1491 Tot $=$

2.4201

Oscillator strength, $\mathrm{f}=\quad 0.0672$

Restricted Singlet Excited State 9:

Excitation energy $=0.1125123634$ hartrees $\quad 3.06161718 \mathrm{eV}$ $404.96 \mathrm{~nm}$

\begin{tabular}{|c|c|c|}
\hline \multicolumn{2}{|c|}{ excitation } & X coeff. \\
\hline 216 & 22 & -0.176 \\
\hline $218=$ & 227 & -0.94346 \\
\hline $226=>$ & 228 & 0.22 \\
\hline
\end{tabular}


Transition dipole moment (debye):

$\mathrm{X}=-0.1027 \quad \mathrm{Y}=0.1152 \quad \mathrm{Z}=-0.0096 \quad$ Tot $=$ 0.1547

Oscillator strength, $\mathrm{f}=\quad 0.0003$

Restricted Singlet Excited State 10:

Excitation energy $=0.1165207106$ hartrees

$3.17068986 \mathrm{eV}$ $391.03 \mathrm{~nm}$

excitation $\mathrm{X}$ coeff.

$217=227 \quad 0.97537$

Transition dipole moment (debye):

1.2194

$\mathrm{X}=-0.5734 \quad \mathrm{Y}=-0.0893 \quad \mathrm{Z}=1.0725 \mathrm{Tot}=$

Oscillator strength, $\mathrm{f}=\quad 0.0179$

Triplet calculation:

Total energy:

-2793.58112271530 hartrees

Final geometry:

$\begin{array}{lccc} & & \text { angstroms } & \\ \text { atom } & \mathrm{x} & \mathrm{y} & \mathrm{z} \\ \mathrm{N} 1 & -0.0034165867 & 0.1342997949 & -0.0004370497 \\ \mathrm{C} 2 & -0.0009498974 & 0.1245143224 & 1.4004970137 \\ \mathrm{C} 3 & 1.3210423977 & 0.0652672554 & 2.0621262937 \\ \mathrm{C} 4 & 2.4888478386 & 0.2828051282 & 1.2753380302 \\ \mathrm{C} 5 & 2.4411066940 & 0.2761529004 & -0.1479328825 \\ \mathrm{C} 6 & 1.1202488305 & 0.3388978666 & -0.8131769903 \\ \mathrm{C} 7 & 3.7074775052 & 0.6538484654 & 1.9270751032 \\ \mathrm{C} 8 & 3.7323534725 & 0.8024898702 & 3.3373361415 \\ \mathrm{C} 9 & 2.6441185914 & 0.2583569968 & 4.0778945376 \\ \mathrm{C} 10 & 1.4365751447 & -0.0697100887 & 3.4877018862 \\ \mathrm{C} 11 & 4.8419557160 & 1.0024257935 & 1.1512329443 \\ \mathrm{C} 12 & 5.8409891554 & 1.8428756752 & 1.7354995190 \\ \mathrm{C} 13 & 5.6559182277 & 2.2972955872 & 3.0663717059 \\ \mathrm{C} 14 & 4.7275624952 & 1.6485720336 & 3.9205773234 \\ \mathrm{C} 15 & 3.6607354689 & 0.3411164082 & -0.9028341830 \\ \mathrm{C} 16 & 4.8280501280 & 0.6575070903 & -0.2305373768 \\ \mathrm{C} 17 & 6.3076509739 & 3.4939744489 & 3.5041634901 \\ \mathrm{C} 18 & 7.1367220906 & 4.2062226556 & 2.5901993013 \\ \mathrm{C} 19 & 7.5315831028 & 3.5890505560 & 1.3540101269\end{array}$




\begin{tabular}{|c|c|c|c|}
\hline $\mathrm{C} 20$ & 6.9109811269 & 2.4082689300 & 0.9869644958 \\
\hline $\mathrm{C} 21$ & 6.0015754568 & 4.0144995534 & 4.7958179699 \\
\hline $\mathrm{C} 22$ & 6.3061592771 & 5.4345257413 & 5.0781771843 \\
\hline $\mathrm{N} 23$ & 7.1271023989 & 6.0958398333 & 4.1557352409 \\
\hline $\mathrm{C} 24$ & 7.4638605944 & 5.6197839887 & 2.8813722322 \\
\hline $\mathrm{C} 25$ & 5.2873772399 & 3.2047059162 & 5.7438890674 \\
\hline $\mathrm{C} 26$ & 4.7136758572 & 2.0285103480 & 5.2932342736 \\
\hline $\mathrm{C} 27$ & 0.3202096121 & -0.5726359712 & 4.3503929942 \\
\hline $\mathrm{C} 28$ & -0.3561354934 & -1.7340005674 & 3.9420951755 \\
\hline $\mathrm{C} 29$ & -1.3808295723 & -2.2930238874 & 4.6926405927 \\
\hline $\mathrm{C} 30$ & -1.7643559825 & -1.6890197466 & 5.8889750721 \\
\hline $\mathrm{C} 31$ & -1.0862646756 & -0.5593463972 & 6.3307647184 \\
\hline $\mathrm{C} 32$ & -0.0338336400 & 0.0113832286 & 5.5953062728 \\
\hline $\mathrm{C} 33$ & 3.7212171550 & 0.0505053573 & -2.3700618370 \\
\hline $\mathrm{C} 34$ & 4.4629792476 & 0.8294812690 & -3.2963772278 \\
\hline $\mathrm{C} 35$ & 4.4622484606 & 0.4466476295 & -4.6481092705 \\
\hline $\mathrm{C} 36$ & 3.7844838752 & -0.6822231345 & -5.0926842244 \\
\hline $\mathrm{C} 37$ & 3.1007804807 & -1.4763405137 & -4.1734052234 \\
\hline $\mathrm{C} 38$ & 3.0729416565 & -1.1043600208 & -2.8368207403 \\
\hline C39 & 8.6030173572 & 4.1421226223 & 0.4672991605 \\
\hline $\mathrm{C} 40$ & 9.8128011801 & 4.5293628408 & 1.0660749880 \\
\hline $\mathrm{C} 41$ & 10.8790527263 & 5.0180138795 & 0.3251259945 \\
\hline $\mathrm{C} 42$ & 10.7540513558 & 5.1406173618 & -1.0577685787 \\
\hline $\mathrm{C} 43$ & 9.5795194013 & 4.7296418533 & -1.6762248890 \\
\hline $\mathrm{C} 44$ & 8.4955111148 & 4.2109052545 & -0.9475668024 \\
\hline $\mathrm{C} 45$ & 5.1722520676 & 3.5592755049 & 7.1948575402 \\
\hline $\mathrm{C} 46$ & 3.9705138696 & 3.4325087905 & 7.9414079981 \\
\hline $\mathrm{C} 47$ & 3.9890953440 & 3.7755077884 & 9.3040560119 \\
\hline $\mathrm{C} 48$ & 5.1489195366 & 4.2002403331 & 9.9417939307 \\
\hline C49 & 6.3410268429 & 4.2709837926 & 9.2218770302 \\
\hline $\mathrm{C} 50$ & 6.3389606378 & 3.9570543286 & 7.8695627504 \\
\hline O51 & -1.0646076200 & 0.2148161128 & 2.0052496303 \\
\hline $\mathrm{O} 52$ & 0.9667885440 & 0.6047442035 & -2.0008852089 \\
\hline $\mathrm{O} 53$ & 7.9586341094 & 6.3882058635 & 2.0633368634 \\
\hline O54 & 5.8468488149 & 6.0545165541 & 6.0319413610 \\
\hline C55 & 0.7561017700 & 1.0953323572 & 6.2558851183 \\
\hline $\mathrm{C} 56$ & 1.3380183482 & 0.7892648906 & 7.4961997487 \\
\hline $\mathrm{C} 57$ & 2.3027599965 & 1.6158523490 & 8.0616649610 \\
\hline $\mathrm{C} 58$ & 2.7284691998 & 2.7857490906 & 7.4124335003 \\
\hline C59 & 2.0349393301 & 3.1716146514 & 6.2535888306 \\
\hline C60 & 1.0661134736 & 2.3411450529 & 5.6856669457 \\
\hline C61 & 7.3753958734 & 3.5998127658 & -1.7304766019 \\
\hline C62 & 7.7204346834 & 2.5726728405 & -2.6242562483 \\
\hline C63 & 6.7497565917 & 1.7569369797 & -3.1953345305 \\
\hline
\end{tabular}




\begin{tabular}{|c|c|c|c|}
\hline C64 & 5.3892385354 & 1.9316858797 & -2.8939225558 \\
\hline C65 & 5.0335553163 & 3.0542142904 & -2.1279335547 \\
\hline C66 & 6.0089775166 & 3.8742630967 & -1.5562940369 \\
\hline C67 & 7.4275170698 & 7.5040361543 & 4.4329134795 \\
\hline C68 & -1.3149690887 & 0.2149123554 & -0.6514005753 \\
\hline H69 & 2.7399368993 & 0.1540967880 & 5.1480596150 \\
\hline $\mathrm{H} 70$ & 5.7498028917 & 0.7036039577 & -0.7903684254 \\
\hline H71 & 7.2106131014 & 1.9337672576 & 0.0649039101 \\
\hline $\mathrm{H} 72$ & 4.1845275152 & 1.4084822158 & 6.0007520765 \\
\hline H73 & -0.0609808587 & -2.2088356412 & 3.0120630269 \\
\hline H74 & 9.9134794180 & 4.4361577303 & 2.1425646054 \\
\hline $\mathrm{H} 75$ & -1.8795388843 & -3.1921967877 & 4.3433956785 \\
\hline H76 & 11.7992685288 & 5.3054036007 & 0.8248949455 \\
\hline H77 & -2.5757872930 & -2.1023214193 & 6.4809078690 \\
\hline $\mathrm{H} 78$ & 11.5709742286 & 5.5383287116 & -1.6527026269 \\
\hline H79 & -1.3667597548 & -0.0973061137 & 7.2728043498 \\
\hline H80 & 9.4874739572 & 4.8003258914 & -2.7559949108 \\
\hline H81 & 1.0965148781 & -0.1547675788 & 7.9757129407 \\
\hline H82 & 8.7698079678 & 2.3544697304 & -2.7985801642 \\
\hline H83 & 2.8020127256 & 1.3058114025 & 8.9748757574 \\
\hline H84 & 7.0530310138 & 0.9127172057 & -3.8070675162 \\
\hline H85 & 2.2997479602 & 4.1006777081 & 5.7577058989 \\
\hline H86 & 3.9849561080 & 3.2507143374 & -1.9253608668 \\
\hline H87 & 0.5888869489 & 2.6353124710 & 4.7556385114 \\
\hline H88 & 5.7067942402 & 4.6988754836 & -0.9176071000 \\
\hline H89 & 3.0642219143 & 3.6957138615 & 9.8679154567 \\
\hline H90 & 5.0159931256 & 1.0557994985 & -5.3564794312 \\
\hline H91 & 5.1244469578 & 4.4582472539 & 10.9965513161 \\
\hline H92 & 3.8030079185 & -0.9478853642 & -6.1455798541 \\
\hline H93 & 7.2641026432 & 4.5714022529 & 9.7080965333 \\
\hline H94 & 2.5884175638 & -2.3779573238 & -4.4954566025 \\
\hline H95 & 7.2684251074 & 4.0115853479 & 7.3120578592 \\
\hline H96 & 2.5396810611 & -1.7254411006 & -2.1242821004 \\
\hline H97 & -1.2145419926 & -0.1603870941 & -1.6672977532 \\
\hline H98 & -2.0215916091 & -0.3770680555 & -0.0731142436 \\
\hline H99 & -1.6718100117 & 1.2488211459 & -0.6888974073 \\
\hline H100 & 7.5040863337 & 7.6305826249 & 5.5107274690 \\
\hline H101 & 8.3586926336 & 7.7600391486 & 3.9319761181 \\
\hline H102 & 6.6335885893 & 8.1564966321 & 4.0565525183 \\
\hline
\end{tabular}

\section{(2) Crystal Data}

Bond precision: $\quad \mathrm{C}-\mathrm{C}=0.0047 \mathrm{~A} \quad$ Wavelength $=1.54184$ 
Cell: $\quad a=15.6203(2) \quad b=19.0603(2) \quad c=20.6701(3)$

alpha $=84.259(1) \quad$ beta $=86.617(1) \quad$ gamma $=77.197(1)$

Temperature: $100 \mathrm{~K}$

$\begin{array}{llc} & \text { Calculated } & \text { Reported } \\ \text { Volume } & 5966.65(13) & 5966.65(13) \\ \text { Space group } & \text { P }-1 & \text { P }-1 \\ \text { Hall group } & \text {-P } 1 & -\mathrm{P} 1\end{array}$

Moiety formula

2(C70 H50 N2 O4), C H Cl3, 3(C2 H3 N) [+ solvent] C H Cl3, 2(C70 H50 N2 O4),3(C2 H3 N)

Sum formula C147 H110 Cl3 N7 O8 [+solvent] C147 H110 Cl3 N7 O8

$\mathrm{Mr}$

2208.77

2208.76

Dx,g cm-3

1.229

1.229

Z

2

2

$\mathrm{Mu}(\mathrm{mm}-1)$

1.195

1.195

F000

2312.0

2312.0

F000'

2320.41

h,k, lmax

$19,23,25$

$19,23,25$

Nref

23835

23698

Tmin,Tmax

$0.806,0.887$

$0.767,1.000$

Tmin'

0.787

Correction method $=$ \# Reported $\quad \mathrm{T} \quad$ Limits: $\mathrm{Tmin}=0.767 \quad \mathrm{Tmax}=1.000$

AbsCorr $=$ MULTI-SCAN

Data completeness $=0.994$

$\operatorname{Theta}(\max )=72.974$

$\mathrm{R}($ reflections $)=0.1241(20581)$

wR2(reflections $)=0.4151(23698)$

$\mathrm{S}=1.949$

Npar $=1449$

1. Y. Zhong, B. Kumar, S. Oh, M. T. Trinh, Y. Wu, K. Elbert, P. Li, X. Zhu, S. Xiao, F. Ng, M. L. Steigerwald and C. Nuckolls, J. Am. Chem. Soc., 2014, 136, 81228130.

2. N. V. Handa, K. D. Mendoza and L. D. Shirtcliff, Org. Lett., 2011, 13, 47244727.

3. V. Percec, E. Aqad, M. Peterca, M. R. Imam, M. Glodde, T. K. Bera, Y. Miura, V. S. K. Balagurusamy, P. C. Ewbank, F. Würthner and P. A. Heiney, Chem.Eur. J., 2007, 13, 3330-3345.

4. S. Erten-Ela and G. Turkmen, Renewable Energy, 2011, 36, 1821-1825.

5. Y. Huang, L. Fu, W. Zou, F. Zhang and Z. Wei, The Journal of Physical Chemistry C, 2011, 115, 10399-10404.

6. A. Velian, S. Lin, A. J. M. Miller, M. W. Day and T. Agapie, J. Am. Chem. Soc., 2010, 132, 6296-6297.

7. T. Teraoka, S. Hiroto and H. Shinokubo, Org. Lett., 2011, 13, 2532-2535. 
